\title{
DIAFIT: DESIGNING CUSTOMIZABLE WEARABLES FOR TYPE 1 DIABETES MONITORING
}

by

Erman Akyol

Submitted to OCAD University in partial fulfillment of the requirements for the degree of Master of Design in Inclusive Design Toronto, Ontario, Canada, 2020 


\title{
DiaFit: Designing customizable wearables for type 1 diabetes monitoring
}

\begin{abstract}
As the need for customization of medical devices has become increasingly clear, designers have responded to the challenge. Despite extensive literature on the importance of customization, few studies have examined its impact on such wearable devices as glucose monitors. Little is known about how user experience and situational context may influence how diabetics choose these technologies and adapt them to manage their conditions. In this paper, we present a co-design study to learn about people's use and the possibility of them building their own T1D glucose monitors, a device that is used on a daily basis to support everyday care. In the first phase, we interviewed and conducted brainstorming sessions to identify the strengths and challenges of current T1D glucose monitors and identified ideas for improving the monitor design. Our first phase revealed that users face three main problems with their monitors: they pose a physical hindrance, do not provide privacy, and are not aesthetically pleasing. Our brainstorming sessions highlighted that to address some of these concerns, customization was key. Informed by our data and literature, in the second phase, we built and evaluated our prototype system, DiaFit, consisting of interactive physical modules for building customizable T1D monitors. Our prototype consists of multiple modular accessories that people can assemble together to build a monitor which can be worn on the wrist, finger, ear or neck. The modules provide visual, audio and haptic feedback about the glucose levels. Our evaluation shows that participants could build their own glucose monitors using DiaFit and found these devices could better support their need for form, privacy, and sense of aesthetics. Users could create combinations of feedback and different accessories depending on the situational context. In particular, adults who self-identified as makers were particularly interested in using DiaFit and building different versions of the monitors more often. We found that customization and a do-it-yourself (DIY) approach could prove useful in the development of inclusive designs for T1D. Our main contribution is our design and evaluation of a prototype system for building personalized wearable T1D monitors using a variety of modules and accessories in a DIY approach. We conclude by discussing lessons learned and outline implications for future researchers interested in exploring bespoke DIY solutions for medical devices.
\end{abstract}


$\begin{array}{ll}\text { Abstract } & 1\end{array}$

LIST OF FIGURES $\quad 4$

$\begin{array}{lc}\text { LIST OF TABLES } & 5\end{array}$

$\begin{array}{lc}\text { 1. Introduction } & 6\end{array}$

$\begin{array}{ll}\text { 1.4. Overall Methodology } & 7\end{array}$

2.1. Current Technologies for T1D 9

2.2 Challenges with Technology for T1D 11

2.3. Customizable Medical Technologies 12

2.3. Customizable Technologies for T1D 12

3. Co-Design Study 14

3.1. Methodology and Procedure 14

3.2. Participants $\quad 15$

3.3. Data Sources and Analysis 15

3.4. Co-Design Study Results 16

3.4.1. Portability 16

3.4.2. Feedback 18

3.4.3. Privacy 20

4. DiaFit's Design and Implementation 21

$\begin{array}{ll}\text { 4.1. Design } & 22\end{array}$

4.1.2. Feedback Modules 24

4.1.3. Implementation: $\quad 25$

$\begin{array}{ll}5.1 \text { Procedure } & 27\end{array}$

$\begin{array}{ll}5.2 \text { Participants } & 27\end{array}$

$\begin{array}{ll}\text { 5.3 Data Source and Analysis } & 27\end{array}$

6. Results $\quad 28$

6.1 Building Multiple Glucose Monitor Configurations 28

$\begin{array}{ll}6.1 .1 \text { Wristband } & 30\end{array}$

6.1.2. Ring 30

6.1.3. Earpiece 30

6.1.4. Necklace 31

6.2. Situational contexts 31

6.2.1. Indoor activities $\quad 31$

6.2.2. Outdoor activities 32

6.2.3. Workplace context 32 
6.2.4. Social context 33

6.2.5. Casual private context 33

6.3. Frequency of customization 33

6.4. Suggestions for Future 33

6.5. Heuristic evaluation $\quad 35$

6.6. Task analysis (TLX): $\quad 36$

7. Discussion $\quad 37$

7.1. Individual experiences and differences $\quad 37$

7.2. Customization and DIY 39

$\begin{array}{ll}\text { 7.3. Limitations } & 40\end{array}$

8. Conclusion 42

8.1. Future Work $\quad 42$

8.1.1. Future research opportunities 42

8.2.2. Future design opportunities $\quad 42$

$\begin{array}{ll}\text { Appendix A - Sketches } & 50\end{array}$

Appendix B - Recruitment Poster 51

Appendix C - Heuristics Evaluation and Task Analysis of DiaFit 52 


\section{LIST OF FIGURES}

$\begin{array}{lr}\text { Figure 1. DiaFit, its accessories } & 8\end{array}$

Figure 2. DiaFit could be worn on the wrist, finger, ear and neck $\quad 8$

$\begin{array}{lr}\text { Figure 3. Examples of BG Meters } & 10\end{array}$

$\begin{array}{ll}\text { Figure 4. Examples of CGMs } & 10\end{array}$

Figure 5. Examples of insulin pumps 11

Figure 6. Artificial Raspberry Pi Pancreas 13

Figure 7. A project of Nightscouts $\quad 13$

Figure 8. Two of the pictive templates and materials 16

Figure 9. Physical wearable templates 16

Figure 10. Participants are using the pictive templates and creating their $2 \mathrm{D}$ and 3D prototypes 16

$\begin{array}{ll}\text { Figure 11. P4's earphone prototype } & 18\end{array}$

$\begin{array}{lr}\text { Figure 12. P5's sketches illustrating earphone } & 18\end{array}$

Figure 13. P4's necklace, bracelet and ring ideas 19

Figure 14. P3's bracelet idea $\quad 19$

Figure 15. P5's suggestion of a watch design which provides visual feedback 19

Figure 16. P2's watch idea with holographic feedback $\quad 21$

Figure 17. P1's tooth, patch, watch and bra ideas $\quad 22$

Figure 18. P5's sketches on a patch idea $\quad 22$

Figure 19. P4's ring idea, its prototype and how it can be used. 23

Figure 20. Prototype of DiaFit 24

Figure 21. Wristband 24

Figure 22. Ring $\quad 25$

Figure 23. Earpiece $\quad 25$

Figure 24. Necklace $\quad 25$

Figure 25. Visual feedback module $\quad 26$

Figure 26. Haptic feedback module $\quad 26$

Figure 27. Audio feedback module $\quad 27$

Figure 28. Components of the modules $\quad 27$

Figure 29. Sample configurations 28

Figure 30. Users' interaction with the prototype $\quad 30$

Figure 31. A digital sketch for DiaFit 


\section{LIST OF TABLES}

Table 1. Configurations of modules per accessory $\quad 31$

Table 2. Configurations of modules for each accessories for situational contexts 32

Table 3. Results of heuristic evaluation 37

Table 4. Results of task analysis $\quad 38$ 


\section{Introduction}

People with Type 1 Diabetes (T1D) use medical devices such as glucose pumps and monitors daily. Although such medical devices are designed to be efficient in providing accurate measures, they do not meet the individual needs of the people using them [1]. For example, commercially available budget glucose monitors such as True Metrix are known to have portability issues. They are bulky, heavy to carry, and can hurt the person in scenarios such as playing contact sports [2]. Similarly, the audio-based feedback is often either very loud or too subtle, not offering enough level of control to ensure privacy and choice [37]. Due to these challenges, individuals with T1D and researchers in the area of design for health have suggested and demonstrated the benefits of facilitating customization by building bespoke solutions for medical devices [4]. In this context, customization is defined as a modification of the functionality and presentation of a given product [5]. Customization in general invites individuals to project their personality, values, and preferences onto a product. As a result, the customized product is a true reflection of who and what they are and what they need $[6,7]$.

One solution for reducing the mismatch between product features and user needs is customization, as it allows users to determine the most appropriate product characteristics for themselves instead of just adopting a given product. Personalization (either user or system-initiated) is recommended as one strategy to fight back against problems that hinder engagement with achievement-based personal informatics systems [8]. Similarly, Harrison et al. argue that it is crucial for designers of trackers to facilitate the customization of tracking and social functionalities and avoid unnecessary workarounds and reflect the users' needs and desires for long-term engagement [9]. Despite the recognition of the possibilities of customization to enhance personal engagement with wearables, relatively little is known about how the strategy actually works, is used and even perceived by people with T1D in a real-world setting.

Some current commercial solutions such as Dexcom 6 demonstrate customization. People using Dexcom 6 can choose to send information about their glucose level to either their monitors, smartphone or smartwatch. Furthermore, many of the existing apps for diabetes management enable people to e display a number of diabetes-related data, such as blood glucose readings, carbohydrate intake, and insulin doses $[10,11]$, although, recent reviews have demonstrated that very few of these apps use this information to provide users with personalized feedback, education, or motivation [11, 12]. Clinical guidelines emphasize the importance of customized feedback and targeted education for behavior change and comprehensive self-management [13]. Nightscout (CGM in the Cloud) is one the examples of an open source, DIY project that allows real time access to a CGM data via personal website, smartwatch viewers, or apps and widgets available for smartphones [14]. It was developed by parents of children with Type 1 Diabetes and has continued to be developed, maintained, and supported by volunteers. Other three major DIY projects for diabetes - OpenAPS, AndroidAPS and Loop - are free, open-source algorithms that attempt to reduce the burden of managing diabetes [15].

\subsection{Goals and Research Question}

Building on prior behaviour studies $[2,16,17,18,19,20,21,22]$ and inspired by the commercial solutions $[23,24,25,26,27,28,29]$, our overarching goal is to explore ways that people can customize form and feedback mechanisms for glucose monitors. Our work specifically contributes a do-it-yourself 
(DIY) design which allows people to build customizable T1D monitors to be used in varied situational contexts (e.g., at home, in public, and when playing sports ). Our studies asked people to reflect on this DIY approach and learned about the types of monitors people would like to build, and stopped short of studying our prototype's impact on behaviour change and comprehensive self-management.

\subsection{Motivation}

Diabetics' behaviours, self-care methods and their experience with wearables such as insulin pens and pumps have already been extensively researched $(2,16,30,31)$; however, their interactions with their devices during various situational contexts, physical environment and physical activities have not. This gap provides an opportunity for research and design. Although there are conceptual designs available [26, 27, 28, 29], they are not supported by research, not implemented or studied. However, there is some research on the use of wearables by non-diabetics during running, cycling [32] and swimming [33].

\subsection{Scope}

We are focusing on designing a continuous glucose monitor for this research. These devices are already known as wearables and carried in various forms on the body, in pockets or bags. Informed by such an understanding, we want to build new wearables to better support varying situational contexts, such as indoor and outdoor activities and social contexts. Wearable technology or devices are smart electronic devices that can be woven into clothing or worn on the body as accessories, thereby making their usage more comfortable. MRP does not propose to build new medical devices, i.e., we will not build a new insulin pump. Instead, we will use existing T1D devices and build new wearables that incorporate these devices and focus on providing monitored information via multisensory feedback mechanisms and enable control of the T1D devices by the people.

\subsection{Overall Methodology}

The constructivist research paradigm applied in this MRP combines several methods for eliciting users' experiences and requirements [68]. The predominantly qualitative methods used in this research fall under the umbrella of co-design [69].

We consider our whole process and methods as co-designing [34]. We have done a deep literature review and an environmental scan, conducted interviews and brainstorm sessions with participants. After analyzing the data we developed a working prototype and evaluated it with the participants. We conducted two-phase meetings with participants to learn about people's use and the possibility of them building their own T1D glucose monitor. In the first phase, We conducted early interviews and brainstorming sessions with five individuals with T1D and learned about how they envision the monitors should look and function. Informed by our results, in the second phase, we outlined a set of design goals for customizable T1D devices and built our prototype system, DiaFit, based on those goals. DiaFit is our design concept which was built as a selfcare wearable monitoring device with multiple accessories and feedback modules (Figure 1). Its accessories can be worn on the wrist, finger, ear or neck (Figure 2). It also has modules for various forms of feedback: visual, audio and haptic. Users could create combinations of feedback on different accessories depending on the situational context. We evaluated DiaFit with five individuals using a methodology inspired by the design critique method [15]. Participants built a number of glucose monitor configurations using our customizable design, and later gave us feedback on our design decisions. Our three key takeaways are: (a) Individual experiences and 
differences affect our choices of T1D medical devices and how we adopt and use them. b) Adults with T1D have a need for flexible and optional use of these devices for various situational contexts. c) Customization and DIY approaches have implications for the design and evaluation of current and future wearable medical devices used in the self-management of diabetes. .

The remainder of this MRP is organized as follows: in chapter 2, we discuss background and related works in the area of medical and customizable technologies for T1D. Chapter 3 describes our early interview and brainstorming study. Chapter 4 describes the design of our prototype and details implementation. Chapter 6 discusses results from the user evaluation. Finally, Chapter 6 includes a discussion of lessons learned and future work.

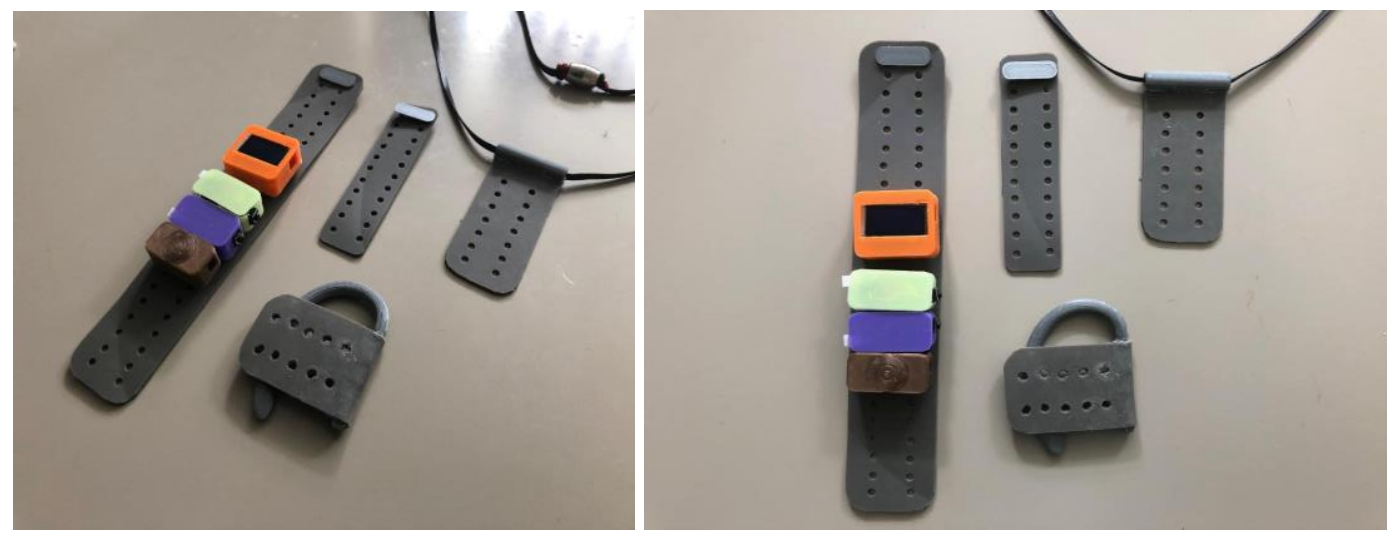

Figure 1. DiaFit, its accessories
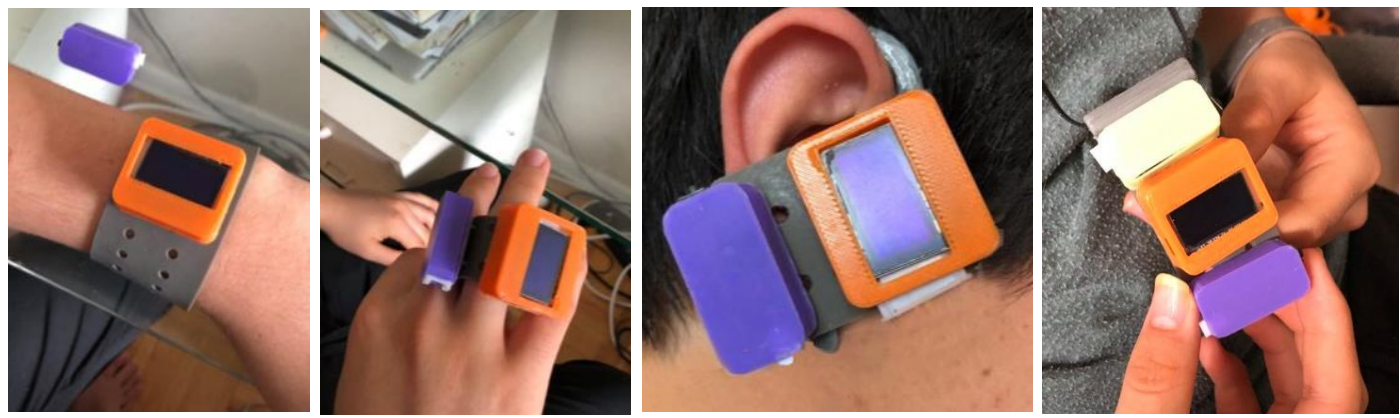

Figure 2. DiaFit could be worn on the wrist, finger, ear and neck.

\section{Background and Related Work}

Wearable technologies are defined as devices that quantify information in order to give wearers "new knowledge about themselves and their environments" [36]. Some wearable technologies, such as continuous glucose monitors (CGM), are classified as medical devices. These devices perform a specific medical function over a sustained period of time. Some of these functions are biologically necessary for good health and survival, as is in the case of the wearable CGMs discussed below.

This research is inspired by a particular do-it-yourself (DIY) approach practiced in the diabetic community projects such as Nightscout and CGM in the Cloud. The work of these groups involves the development and on-going testing of open source software that is used in insulin pump systems, systems 
that typically consist of an insulin pump, a continuous glucose sensor, receiver, and transmitter, and a smartphone. Our approach is focused on the physical wearable device configurations and feedback modalities rather than developing software and electronics used in these appliances.

\subsection{Current Technologies for T1D}

Self-care in the context of T1D includes calculating medication doses to inject based on factors such as food consumption and current blood glucose (BG) levels, which are often measured with a mobile BG meter. In addition to BG meters (Figure 3), there are various commercially available self-care devices including wearable insulin pumps and continuous glucose monitors (CGM), mobile apps, and other digital tools that continue to become available in the market. The pump, which is about the size of a smartphone, delivers insulin through a small tube that connects the pump to an infusion set, a plastic device with a small needle. Continuous glucose monitors (Figure 4), a newer technology, can help reduce the feeling of "flying blind" between the finger checks with blood glucose monitors and give the user information about how their blood sugar numbers are trending: going down, going up, going down fast, going up fast, or remaining stable. Wearable CGM sensors present a potential revolution in diabetes treatment. Development of CGM technologies and applications stimulates scientific research in areas ranging from medicine to physics, electronics and chemistry.

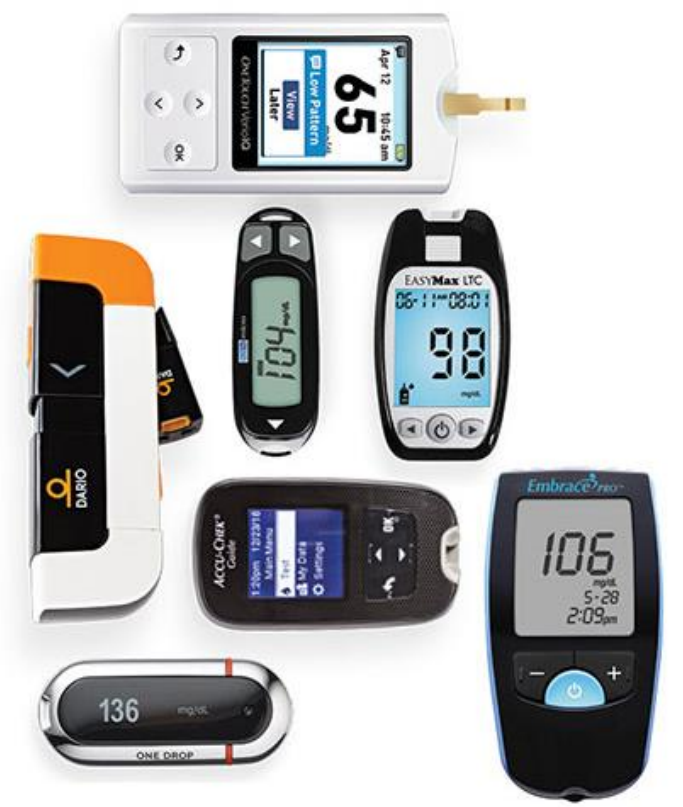

Figure 3. Examples of BG Meters (from: https://bit.ly/34FIKXS) 


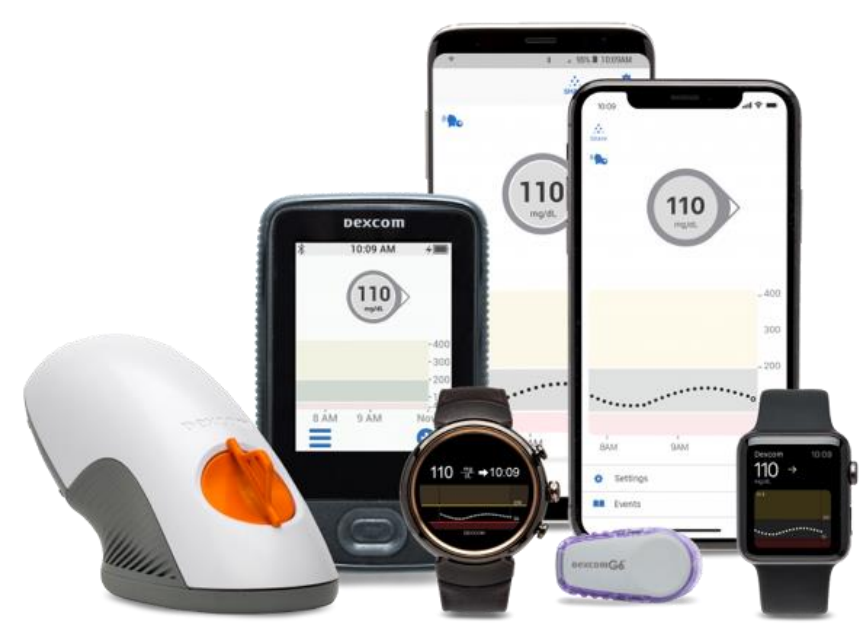

Figure 4. Examples of CGMs (from https://bit.ly/2RGikQo)

Like insulin pumps (Figure 5), stand-alone CGMs are connected to a person's body. A sensor is attached to the body, and a needle in the sensor is inserted under the skin. Through this connection a sensor delivers blood sugar numbers to a receiver every five minutes. A person using this technology can view these numbers in a number of ways: on the receiver itself, on a smartphone, or even on a smartwatch. Many of the newer insulin pumps integrate a CGM. Integrated CGMs work in the same way, but because they are part of the pump system, the CGM transmits data directly to the pump rather than a separate receiver.
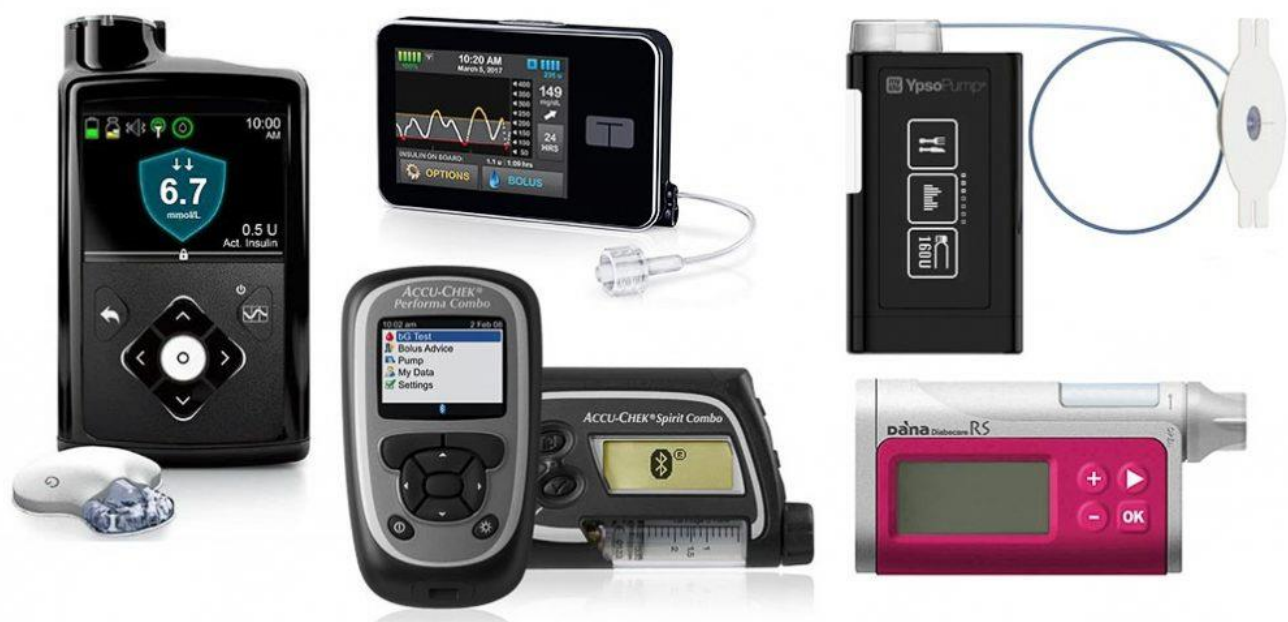

Figure 5. Examples of insulin pumps (from https://bit.ly/2KdRmM0)

As Teston [37] pointed out in her discussion of mHealth technologies (devices and apps that track fitness), many regard the widespread use of cellular phones and other mobile devices for self-tracking health as revolutionary [38]. Apple-watch also provides blood glucose levels after synchronizing with the patient's transmitter. Within the next five years, there is a high probability of having new ways of monitoring blood sugar that require zero finger pricks, injections, or other invasive procedures. One of 
them is wearable technology. Luca et al. (2018) [39] describe significant advances in reading BG (and other diagnostic) levels through the skin surface in their article in Nature. Google Life Sciences/Verily has been developing a smart contact lens. By using tears or the membrane surface of the eye, this contact lens would theoretically be able to read blood glucose. According to an article in Natural News [40] sweat is another option for BGM.

\subsection{Challenges with Technology for T1D}

Adults with T1D are adopting, carrying, and using devices in varied and individualized ways to suit their everyday lives [41], and demonstrated a need and desire for bespoke and personalized self-care devices [42]. For example, Holubová et. al [43] has suggested that physically active people with T1D tend to conceal that information from others and could benefit from a technology that would not represent an obstacle during physical activity and would not call attention to itself.

Some work has shown that CGM may be very useful in certain exercise situations in which vigilant selfmonitoring of blood glucose (SMBG) is just not practical. For example, a team of cyclists with type 1 diabetes used the FreeStyle Navigator to monitor their glucose continuously during their race across America [44]. In one of the best examples of the utility of CGM, the Medtronic Guardian was shown to be very useful for scuba diving when symptoms of hypoglycemia may be masked and SMBG is impossible [45]. A majority of the youth in that study found that CGM was helpful in making insulin adjustments and helped to prevent low blood glucose occuring. However, nearly half of the subjects (46\%) felt that CGM interfered with sports and outdoor play [45]. The size and the weight of the CGM, portability and difficulty of monitoring the screen were a few challenges with the technology during playing sports. Monitoring diabetes is never far from the mind of a CGM user who has a bulky device affixed to the body that must be protected from bumping and dislodging [46].

Another potentially important use of CGM for active diabetics is the ability to track and warn of possible hypoglycemia or hyperglycemia [47, 48], and for that reliable and sufficient feedback mechanisms are required. Patients are sometimes frustrated by false positive alarms. This happens because of inaccuracy of the devices and may happen at any time [49]. For example, CGM alters sleep. Although some CGM users felt they could sleep more easily [50, 51], others mentioned more disrupted sleep, likely due to the frequency of both safety and troubleshooting alarms [50]. Using these devices compels a kinaesthetic response from the user (silencing alerts, checking glucose levels, etc.) which may lead to emotional frustration with the device [52].

Healthcare is personal, so privacy can be important to people with diabetes (e.g. [53]). They can perceive stigma from their communities and their workplaces [54]. CGM users may also experience altered perceptions of body image [50]. Perceived stigma can also cause self-consciousness around glucose meter testing and insulin pump use [55, 56], and people often go to great lengths to hide their condition [45]. They do not use or carry their devices, measure glucose level, sometimes turn off their devices or alarms, do not share about their conditions with others and cover their body to hide their devices and sensors. However, the physical profile of the CGM transmitter is difficult to hide under clothes, making diabetes more visible to others [51]. CGM can further lead to social worries about alarming at 
inconvenient times, causing unwanted attention and a sense of being different [51, 52, 57]. Beyond stigma, concerns about discretion even around family and friends can interfere in diabetes self-care practices [54], and strangers can ask awkward questions [55].

\subsection{Customizable Medical Technologies}

Customizable medical devices are not a new concept. Based on the case studies presented in Holubová et al. [58], there is no one-size-fits-all diabetes self-management tool which would fully satisfy the needs of all individuals. According to Delgado et al. [59] customization will be the best way to provide higher levels of quality, functionality, safety and biocompatibility for surgical devices. Researchers interested in designing low cost, customizable, and innovative medical devices can find references to various easily available electronic components as well as design methodologies to integrate those components for a successful design [60]. With the ability to create customizable products tailored to individual patients, as well as software and hardware, the use of 3D printed medical devices has rapidly increased in recent years and is predicted to be used more often in the future [61]. Open-source documentations and DIY culture may give people the ability to customize their medical devices according to their needs and expectations. We hope to make important contributions to this field.

\subsection{Customizable Technologies for T1D}

According to Franke et al. [36], the value of customization can be measured not only by the outcomes but also by the users' contribution to the design process. Customization experience is another perceived value for users too [62]. Through our design efforts, we hope to offer such user experience values for people with T1D.

DIY activities such as hacking, tinkering, repairing and crafting are growing in popularity [63]. Some maker communities are at the forefront of DIY health and wellbeing, including those designing, customizing and manufacturing technologies for Type 1 Diabetes. They also actively disseminate opensource technologies as widely as possible. There is a community of makers developing their own solutions to meet T1D needs. At the lower fidelity end, they can adopt accessories such as luggage tags to carry their devices or order customized stickers or cases to add to their devices. DIY groups such as Nightscout have modified CGMs to upload data to "CGM in the Cloud' and communicate with other devices such as smartphones and smartwatches [64]. There have also been moves to use CGMs and insulin pumps in conjunction with programmable devices such as Raspberry Pi (Figure 6) to develop more advanced T1D technologies [65]. "The bionic pancreas", an excellent TED talk [66] serves as a good introduction to the concept of an artificial pancreas, a Night Scouts' DIY project (Figure 7). 


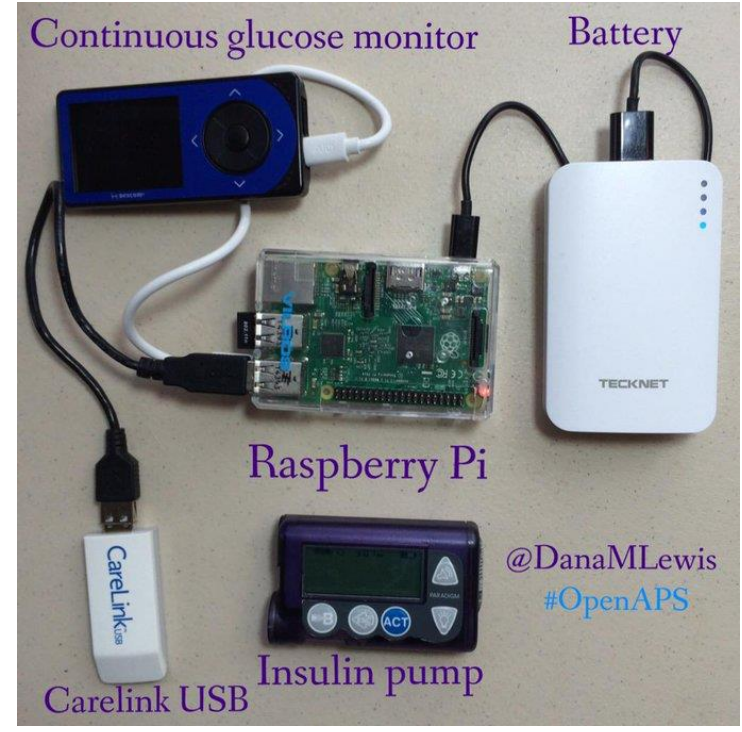

Figure 6. Artificial Raspberry Pi Pancreas.

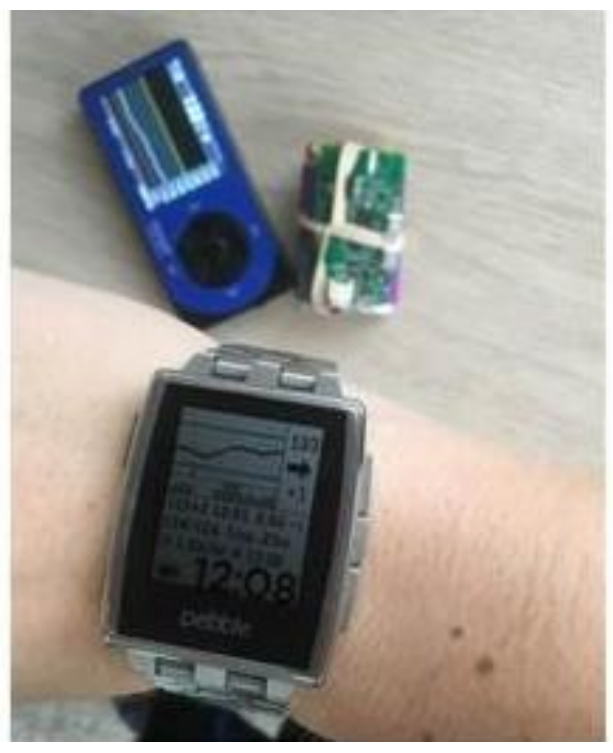

Figure 7. A DIY project of Nightscouts.

Customization is very limited among commercially available CGMs. Only Dexcom 5-6 and Tandem Diabetes Care X:slim provide customizable alerts with a number of different tones to inform users when glucose falls below or rises above user selected limits and when glucose is rising or falling rapidly. [67] The Sensionic CGM's waterproof system uses cell-phones for monitoring and its transmitter provides vibration alerts. Customization can be enhanced with covers, stickers, optional colors and accessories.

Our prototype system offers customization in portability, feedback and aesthetics. We can customize its usage to suit a patient's life-style. Its modularity also allows us to configure and evolve it over time based on changing needs and developments in technology. Although we did not focus on interface design, customization would be possible in interface and visualized data as well in the future iterations. 


\section{Co-Design Study}

The purpose of our co-design session was to convene the participants we are designing for and bring them into the design process. We co-created wearables, investigated how participants want to interact with their devices and examined how they create solutions, and how their solutions function and look like.

\subsection{Methodology and Procedure}

In order to explore our goals (understanding and design), we had a two-part strategy: First, we conducted interviews and brainstormed ideas for wearable devices that meet the needs of people with T1D. Participants were invited to take part in a brainstorming session (40 minutes) based on the PICTIVE method [71, 82]. In this method, participants reflect on design by sketching and creating models using various craft tools or altering an existing researcher created sketch and 3D models [83]. We created a step-by-step procedure for conducting the first part of this research. First, we invited volunteer adult participants to a 1.5 hour interview and brainstorming session. Information about this study was advertised using our flyer and a participant information sheet. If people were interested in participating, they emailed the researcher to book a suitable time. Prior to the study, participants were asked to complete a short (5-6 mins) pre-study questionnaire that collected some basic background information about the participant. They also signed a consent form. On the day of the study session, the participant was briefly reminded of the goals of the project and their rights (20 minutes). Participants next took part in a semi-structured interview (20 mins) where we discussed their past and current positive and negative experiences using T1D medical devices during physical activities (Goal A). Next, the participants were invited to take part in a brainstorming session (40 minutes) based on the PICTIVE method (Goal B). In this activity, the participants were provided with a template drawing sheet (Figure 8) and drawing materials to sketch a few wearable device ideas. In addition to or as an alternative to sketching, participants showcased their prototype ideas using our physical wearable templates (Figure 9). Throughout this session participants were encouraged to think aloud explaining their rationale. Finally, the last 20 minutes were used to discuss the prototypes made (Figure 10). Participants were invited to offer any additional comments and suggest any additional changes to the prototypes. This entire session was videotaped for posterior qualitative and quantitative analysis. The reason for videotaping was to capture both the conversations as well as any interactions the participants demonstrated while explaining their wearable devices. After data was collected, we analyzed the participants' behaviours, problems and suggestions looking for correlations. Participants wanted devices which provided useful, reliable feedback while being inconspicuous and providing minimal interference with their activity. After creating our first working prototype incorporating the participants most frequent requests, we ran another evaluation session. Participants were provided modules which carry different feedback mechanisms (visual, audio, haptic) for their wearables and were asked to customize their wearables considering their needs, as well as how and where on their body they might use it. Participants were given their prototype and asked to build, use and interact with it. These interactions were documented and participants were asked questions, similarly to the co-design session. We later followed up with semi-structured interviews with each participant (average $20 \mathrm{~min}$.), which focused on their experience with the prototype, views on form, wearability and experience of customizability and the DIY approach. 


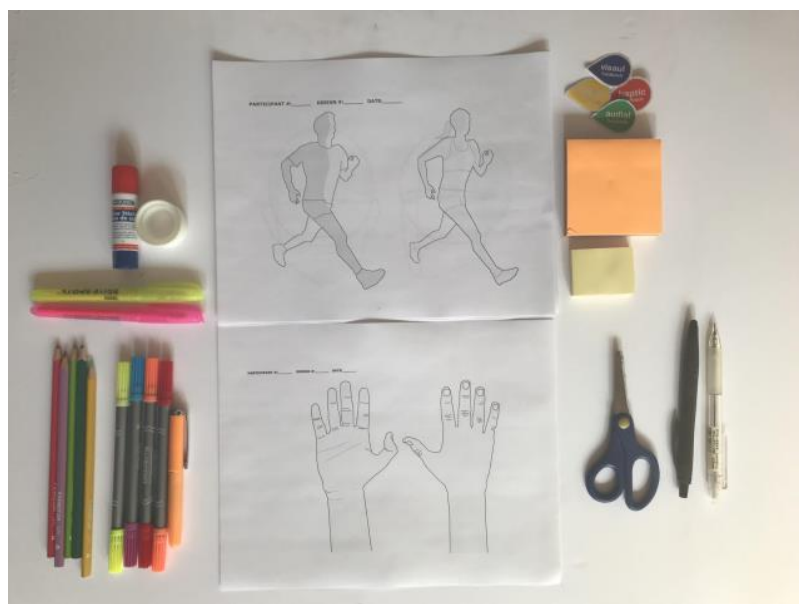

Figure 8. Two of the pictive templates and materials.

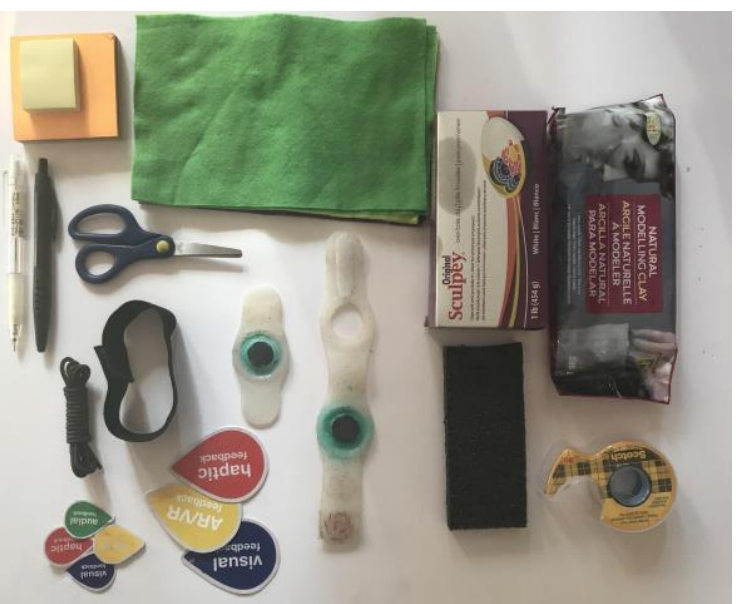

Figure 9. Physical wearable templates.

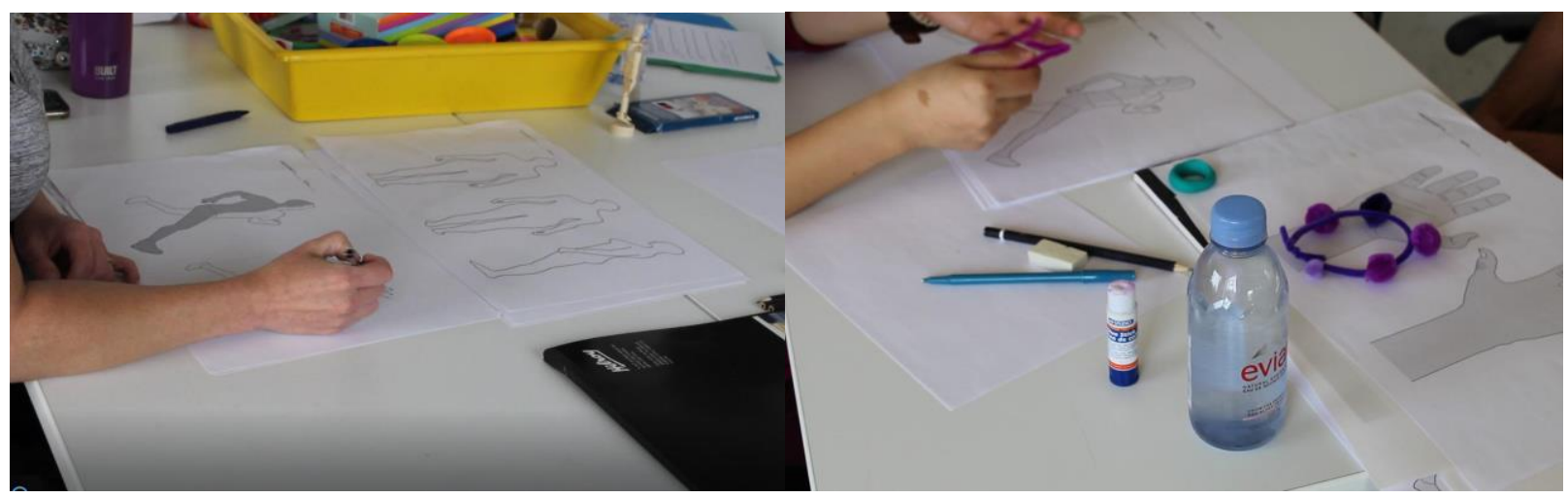

Figure 10. Participants are using the pictive templates and creating their 2D and 3D prototypes.

\subsection{Participants}

Participants were invited on a first-come-first-serve basis for the interview and brainstorming session described above. The participants were all adults ranging between 31 and 56 of age and only one of them was male. They all had T1D and had some past experience of using their T1D devices during physical activities. Two of the participants had 1 year of experience. Others had 3, 15 and 39 years of experience. Regarding participants' experiences with their medical devices during sports and exercise, wwo of the participants were very comfortable, two were comfortable and one was neutral. In this article participants are referred to as "P" and given a number. For example participant one is "P1" and participant number five is "P5".

\subsection{Data Sources and Analysis}

For this research, primary data sources included information collected and processed directly by the researcher, such as semi-structured interviews, and co-design sessions. Secondary data sources included information retrieved through literature reviews and environmental scan. Descriptive analysis [72] was used to summarize individual variables and find common patterns. We first transcribed the recordings verbatim and then used a general inductive method [73] to analyze the transcripts. Transcripts were read repeatedly and text segments coded for potential themes. As the coding framework developed, transcripts 
were reanalyzed for new themes. Fifteen low-fidelity prototypes were created (Fig. 10,11,12,13) during the co-design session and were analyzed. The analysis focused on the elements that influence creating the device, wearability and the usability of the devices. Themes that emerged from the analysis were used to inform the design and development of DiaFit.

\subsection{Co-Design Study Results}

Overall, in line with previous work [27], participants' personal preferences influenced their choice of CGM devices. For example, privacy and the learning curve associated with using new technologies was mentioned as a reason that motivated people to select specific CGM devices. P1 said, "[The monitor] can stay with me. I do not need to go to the washroom, prick my finger, it is just instant and easy. It is a gamechanger for sure." Monitors are provided with CGMs but participants can choose to use them or substitute other wearables or cellphones. Choices were influenced by activity being performed and personal taste. For example, P3 wanted a monitor separated from her phone, because she did not want to take her phone while she is playing sports. She also chose that monitor because it was the smallest and slimmest. P2 was using a watch as a monitor, because it was easy to carry and monitor his BG levels. Cost of the device was also mentioned to be a factor. For example, P4 used her cell-phone because the additional cost of a monitor was too high.

Beyond the results identified in past literature, our examination of participants' responses identified three new findings related to portability, feedback and privacy. These issues are mentioned both in the interviews and co-design sessions. Below we describe the behaviours and problems associated with these three categories of user needs: (1) portability, (2) feedback and (3) privacy.

\subsubsection{Portability}

\section{Behaviour:}

We noted that there are similar behavioral patterns among participants related to carrying devices. For example, Although not all of them carried their monitor in the gym, all participants mentioned that it is more convenient for them to carry their monitors with them when they are working at the gym.

Participants 1, 2 and 4 always carry their monitors while working at the gym. P1 often checks how her glucose changes during activity. P3 does not carry her monitor at her home gym and leaves it in a locker, however, in other gyms she takes her monitor with her as a matter of convenience. P5 leaves her monitor in her locker if the working session is short. She checks her glucose level at the beginning and end of the sessions. P2 wears a watch and monitors his glucose levels regularly, as well as his step counts and heart beat.

\section{Problems:}

There are several problems with carrying a monitor during exercise. First of all, carrying a bulky monitor is awkward. Users carry them in their pockets or bags. If they are using their pockets, their devices weigh down their pants or shorts which ruin their exercising experience. If their monitors are in their bags they must constantly be mindful of the placement of these bags. While P5 uses her cell-phone as a monitor, she 
prefers not to carry it because she does not want to be disturbed while working out. Various problems were also identified related to using monitors during exercise. Body placement could make information retrieval difficult or awkward. For example, P5 observed that during gardening, distance sometimes meant that Bluetooth connection was lost. P1 decided not to take part in Jujitsu lest her device be damaged or ripped off.

\section{Possible solutions:}

To address the above identified problems, participants suggested various ideas for wearables that could be worn on different parts of the body: most common was the wrist (5 of 5), second was the ear (3 of 5), and third one was on a finger ( 3 of 5). All suggested designs were attached to or worn on the body, and not carried in the hands. Most of the participants designed prototypes with non-traditional forms such as rings, bracelets, necklaces. All allowed hands-free monitoring when participating in sports. For example, all participants shared the idea of wearables on the wrist, referring to them as watches (Figure 14 and 15), wristbands or bracelets. P1, P3, andP4's monitor was worn on their ear (Figures 11 and 12), and P3 suggested a device with a separate monitor that could be worn as a necklace (Figure 13). P4's design (Figure 4) could also be adopted to varying contexts, simply functional or more ornamental depending on the occasion. Others opted for the less obtrusive monitors: P1 designed a patch/ plaster-prototype and P3 a prototype to attach to clothing. Overall, our analysis identified that participants wanted more flexibility in how they wear the monitors (Design Goal 1).

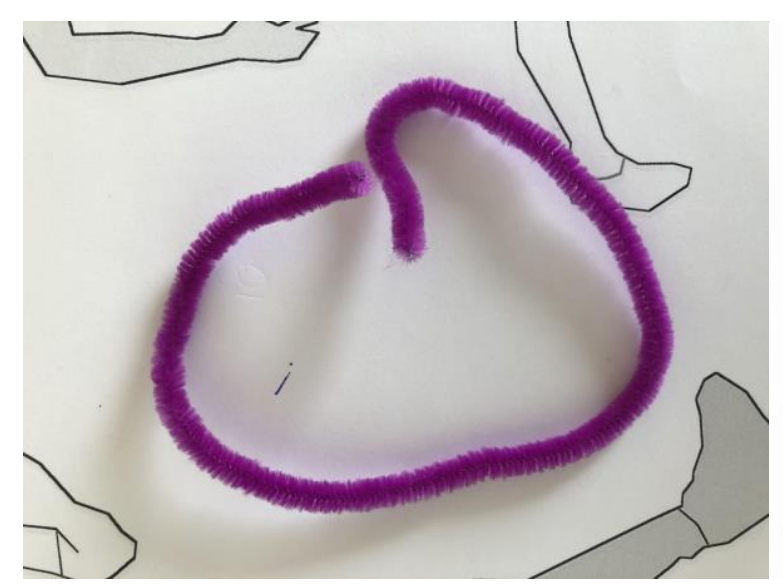

Figure 11. P4's earphone prototype.

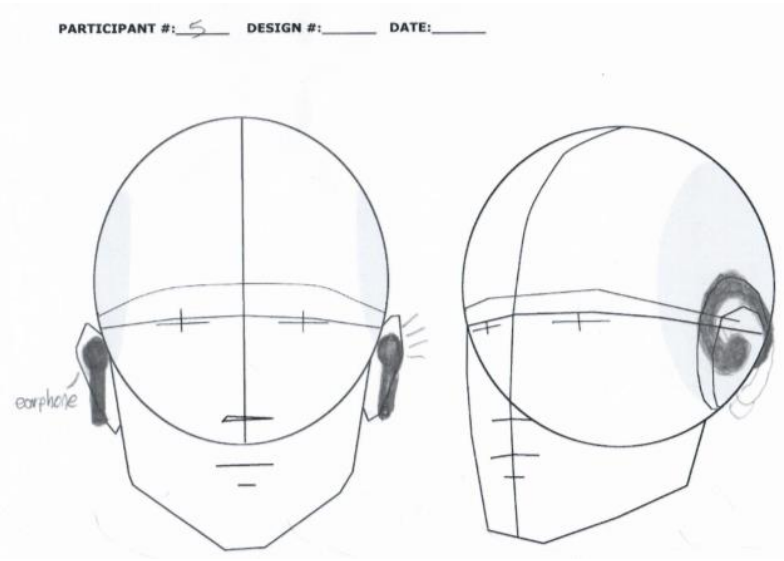

Figure 12. P5's sketches illustrating 


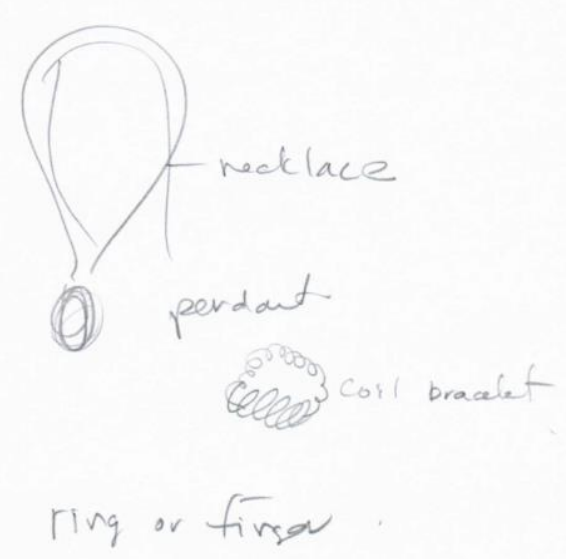

Figure 13. P4's necklace, bracelet and ring ideas

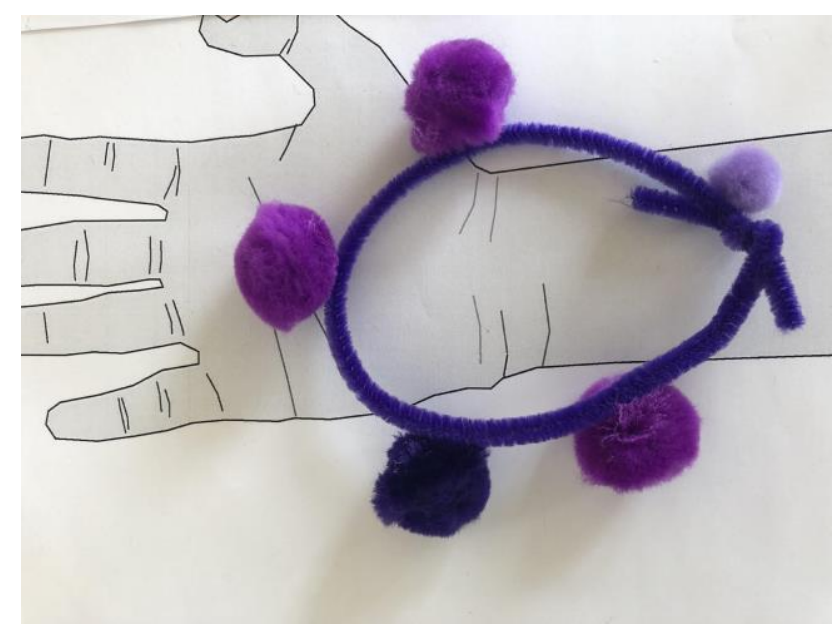

Figure 14. P3's bracelet idea.

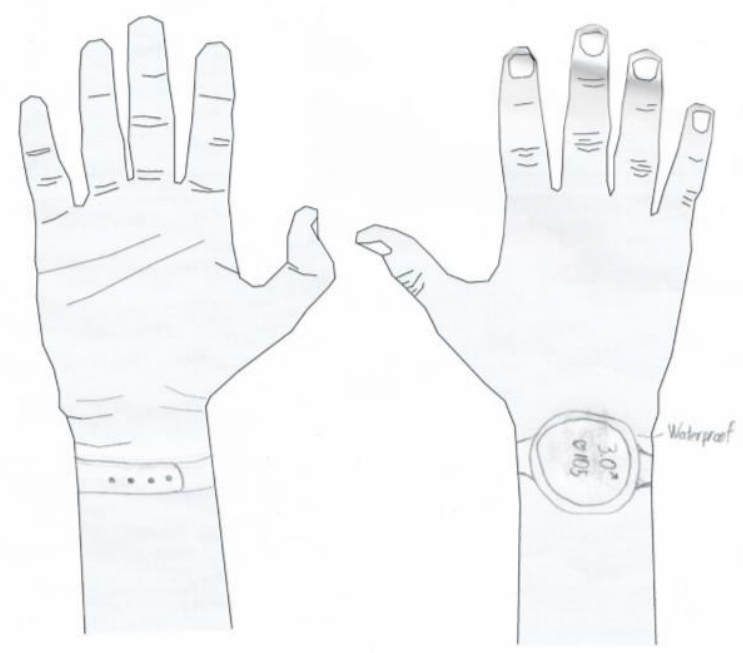

Figure 15. P5's suggestion of a watch design

\subsubsection{Feedback}

\section{Behaviour:}

Participants check the monitor whenever they feel the need (such as, when....). But these feelings alone are not always reliable. Some form of alerting feedback is necessary. In public places, beeping may be embarrassing and participants turn the alarm off or may not wear their devices if they are meeting with strangers. All participants mentioned the embarrassment of beeping in inconvenient times. For example, P3's device started beeping many times because she had low BGL during the interview. Even though she was a very confident participant and open about her condition, I knew about her condition and she said sorry about it many times and said she felt ashamed. It is necessary to check glucose level when an audio alarm is triggered and for that alarm to be heard the device must be within a close range. Although participants do not check their BGL continuously, and leave their monitors in their lockers at the gym by contrast, P1 likes to see how her body reacts while playing sports and keeps the monitor on, watching the changes in numbers and trends. 


\section{Problems:}

Visual and audio feedback are the two main channels used in current commercial solutions. Participants said if they rely on the visual channel solely they might miss crucial information sometimes. They have to check the visual information by themselves without any signal. They said they are mostly aware of the condition of their BGL. Although some of the participants (P2, P3, P4) were usually comfortable talking about their condition and were not bothered by others' comments and staring, they were still selfconscious in unfamiliar circles. Similarly, audio feedback also poses some challenges such as participants may be embarrassed if the audio feedback is loud and gets heard by others. P3 said her monitor sometimes beeps at very inconvenient times, audio feedback is loud and the level of alarm can not be adjusted as she wanted. She said, "I wish I could have the ability to make it go high and low when the monitor beeped. You can not change it for certain high and lows." Contrarily, audio feedback sometimes can not be heard and crucial information is missed if the device is carried in a pocket or a bag. P3 and P5 also said she hears the beeping all the time at home, because she can not carry her device and bluetooth connection does not work all the time at home. P5 said, "I hear it all the time at my house. It won't go through certain walls, right?". When participants are sleeping or drowsy they may not feel their glucose level changing. P1 feels that her body alerts her to glucose level changes while sleeping and that an alarm is unnecessary for her, but is aware that is not the case for others who may be at risk. P4 thought that louder feedback might be important for the elderly or kids who may be distracted while playing or focused on an activity.

\section{Suggested solutions:}

To address the above identified problems, participants suggested using various feedback mechanisms. The most mentioned feedback was haptic (4 of 5) as this would provide discreet and personal feedback to the participants. Participants mentioned that it could be useful, they could feel the haptic feedback when they do not check the monitor or hear the alarm. The second most suggested feedback was audio ( 3 of 5), usually in the form of an ear-piece (Figure 11 and 12). Participants mentioned that audio feedback will enable them to receive personal and discrete feedback and they can continue to receive important information while listening to music or talking to a phone as well. Lastly, visual feedback was mentioned by all participants. All of them currently use a visual display (monitor or cellphone), for monitoring their glucose levels. Information on the monitor is visualized using numbers, an arrow for trend and graphs for changes in glucose levels over a period. In addition to these visualizations, P4 suggested alternatives such as using RGB LEDs in prototypes of bracelets and rings (Figure 13, 14, 19). They reasoned that different colors can give instant clues as to participants' changing glucose levels. For example, if the LED is green then everything is fine and one need not worry about the glucose level at that time. P2's design was more futuristic and her wrist wearable included holographic visual feedback (Figure 16)

Overall, we inferred that people can benefit from having access to different feedback modules which they can either use as a single feedback modality or combine them to build a multimodal device (Design Goal 2). 


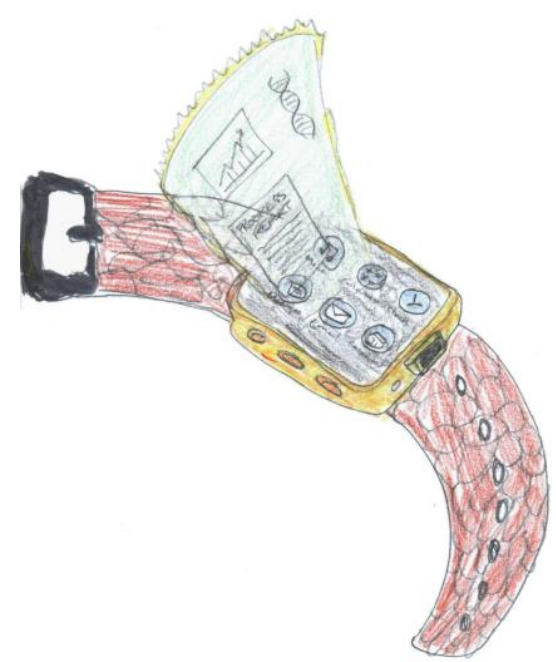

Figure 16. P2's watch idea with holographic feedback.

\subsubsection{Privacy}

Behaviour: The issue of privacy was highlighted by all participants. For example, when P3's monitor started beeping at the beginning of the interview, she said that she usually feels comfortable when it happens among people she knows, but otherwise would feel embarrassed and feel the need to discuss her condition. On the other hand, P1 was not comfortable with people she knew, but felt more comfortable with strangers. P1 suggested the possibility of sharing information with the digital support community but was aware of the problems and privacy issues inherent in sharing personal information too broadly. Overall, users might be private about sharing information with some people or via certain devices. When participants think the data is personal, they also tend to hide their devices.

Problems: The device itself and the feedback it provides compromise privacy. If the alarm beeps, or if their devices are seen by others then they may be questioned or stared at. All participants mentioned that they did not want to deal with awkward questions or receive unsolicited suggestions such as their insulin injections might in fact be an indication of drug addiction. Generally, how their devices looked and their sizes were causes for complaints. Their devices' medical and bulky appearance drew the attention of other people.

Suggested solutions: P1, P3 andP4 wanted their prototypes to be almost invisible. One of P1's design ideas was fitting a device into a tooth (Figure 17), the belly button or between fingers. P1 and P3 had ideas for skin patches (Figures 17 and 18). Both P1 and P4 suggested ideas to embed the sensor's into clothing such as bras (P1, Figure 17) and underwear (P4).

All participants mentioned a wearable on the wrist and the second most common idea was an ear-piece. The rationale for these was they wanted to make the device resemble other common wearables or something that could be hidden under fabric. Three of our participants designed prototypes resembling jewellery (Figures 13, 14 and 19), integrating technology into existing, conventional objects, rendering them "invisible". Participants also highlighted a number of contextual factors that affect privacy 
concerns. While P2, P3 and P5 were fine with their wearable being visible to others, P1 and P4 prefered to hide them in situations where they might be made to feel awkward or self-conscious.

Slimmer, smaller, and less bulky device ideas were also common. All five participants described their monitoring devices as "bulky", and then went on to design elegant, attractive, small prototypes that they considered suitable as casual wear such as P3 designed a necklace (Figure 13) and P4 proposed a fashionable bracelet (Figure 14).

Informed by these suggestions and building on design goals 1 and 2, we think that wearables for T1D can enable people to actively address their privacy concerns arising from form and feedback by providing them with various accessories that enable building different types of monitors for different contexts (Design Goal 3). People can also customize the privacy of their devices by using different feedback modules (facilitated by implementing design goal 2).

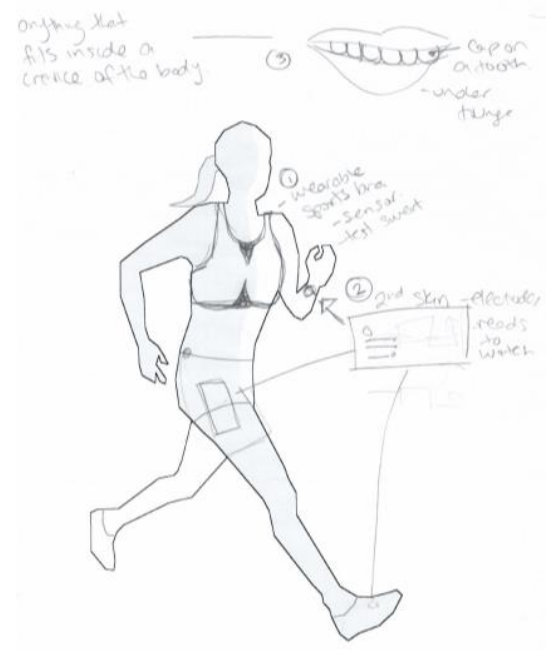

Figure 17. P1's tooth, patch, watch and bra ideas.
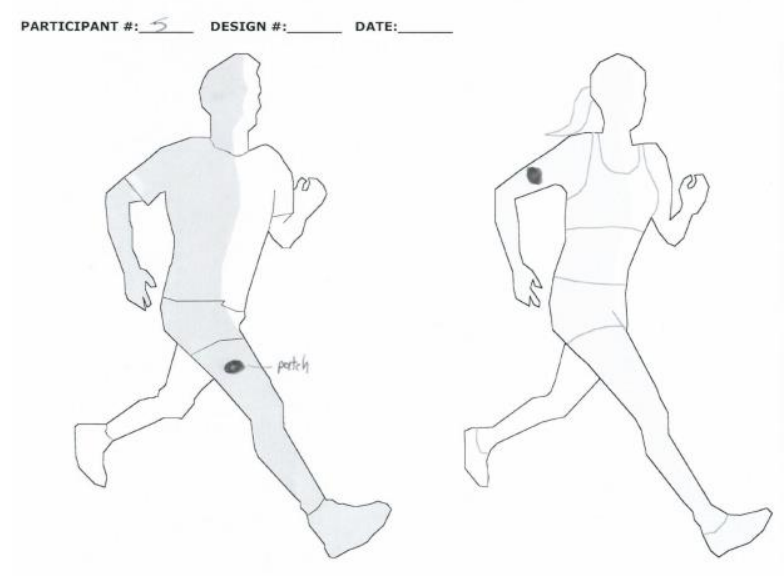

Figure 18. P5's sketches on a patch idea.
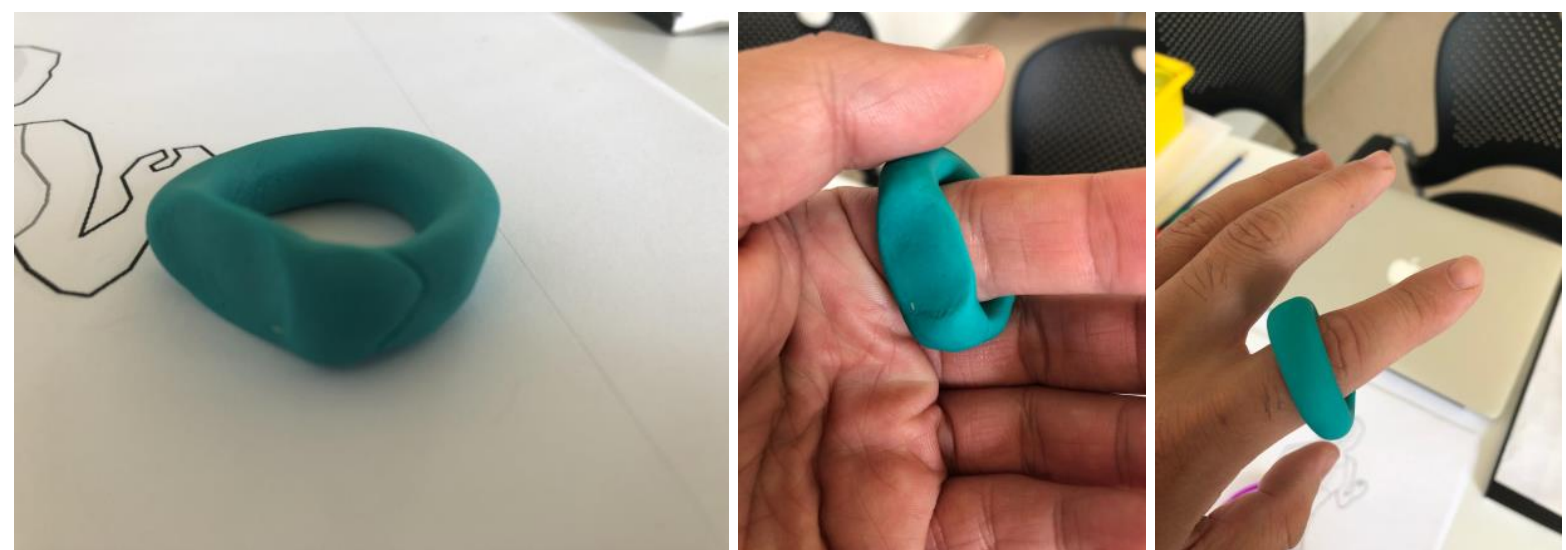

Figure 19. P4's ring idea. 


\section{DiaFit's Design and Implementation}

Participants expressed a belief that the prospect of a customizable wearable device would prove attractive to the T1D community at large. This research might prove to be a valuable starting point in addressing their need for a device offering customizable portability, feedback and privacy (design goals 1-3). Informed by our design goals, we developed a proof-of-concept system, DiaFit, that consists of modular pieces that facilitate the exploration of customization in the context of glucose monitors.

\subsection{Design}

Low and medium fidelity prototypes were created to address our design goals. We brainstormed, sketched several concepts (Appendix A). Our alternative concepts were devices that could be worn in different parts of the body, and could be attached to clothes. Some of our concepts were about modular pieces that users could customize again, wear and use in varying circumstances. We suggested hand free usage and gave privacy to the users. Then, eliminated our options of concepts and finally selected a concept inspired by modular LEGO design to create DiaFit. We created clay models and 3D models of the accessories and feedback modules before 3D printing the final prototypes and also selected and sourced the components. DiaFit is a unique design. It consists of interactive physical modules for building customizable T1D monitors. Our prototype consists of multiple modular accessories (Figure 20) that people can assemble together to build a monitor which can be worn on the wrist, finger, ear or neck. The modules provide visual, audio and haptic feedback about the glucose levels.
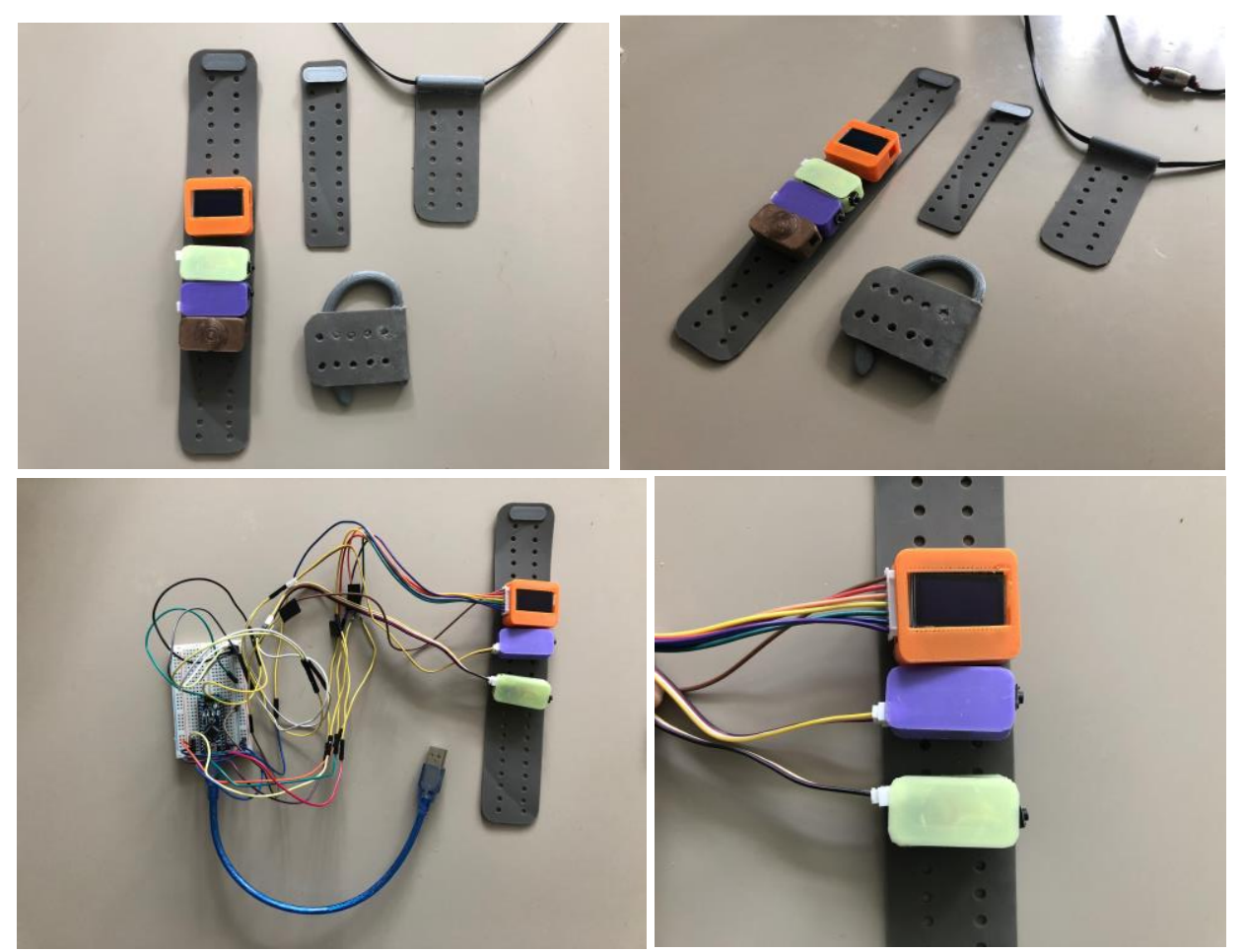

Figure 20. Prototype of DiaFit. 


\subsubsection{Accessories}

Wristband: It looks much like a watch strap. It has holes to attach the modules and snaps. Users can attach the modules anywhere on the strap. It is adjustable and can fit on wrists with various thickness. Rubber was used for the strap and TPE (thermoplastic elastomers) used for the snap (Figure 21, 29).

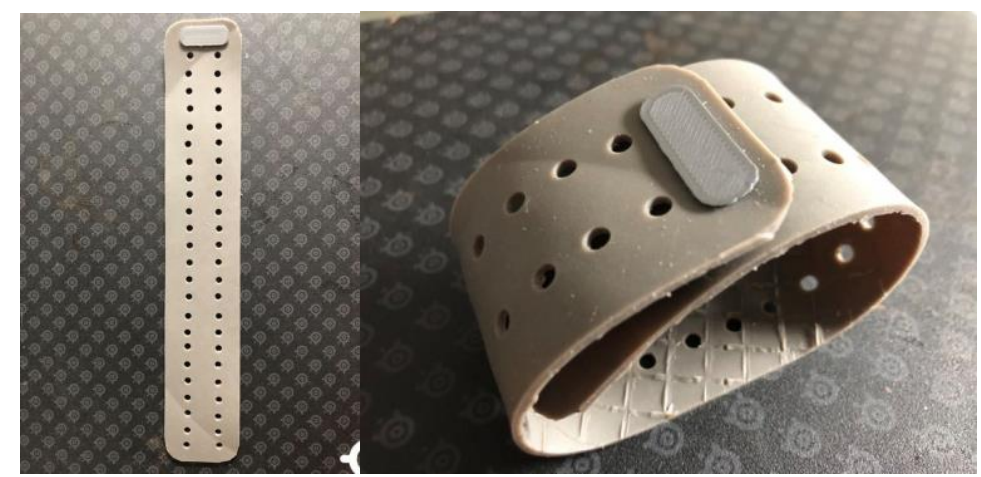

Figure 21. Wristband.

Ring: It has the same features as a wristband. It could be adjustable for different fingers of the same user or users with different finger thickness. Modules could be attached in various orders (Figure 22, 29).

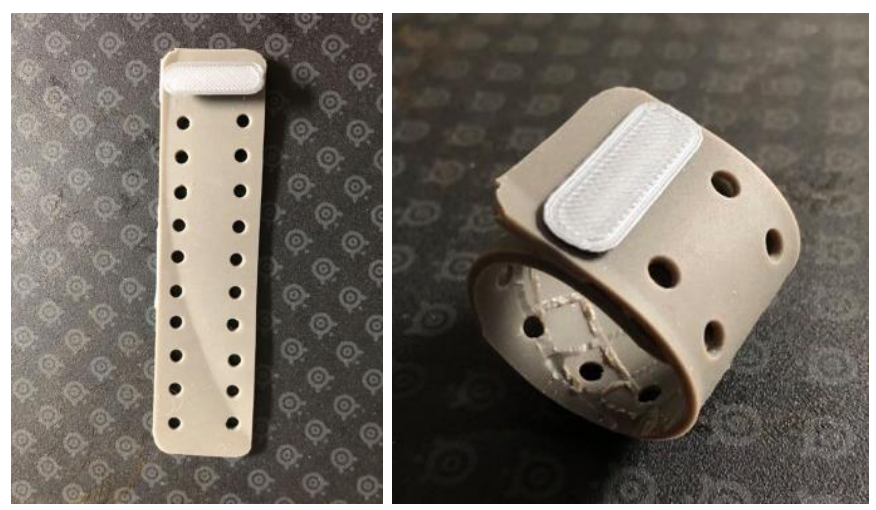

Figure 22. Ring.

Earpiece: This accessory is worn on the ear. The extension goes behind the ear and the other part stays outside of the ear (Figure 22, 29). Outer part has a set of holes to attach the modules. The extension is TPE and the outer part is rubber. Three modules can fit on it. 

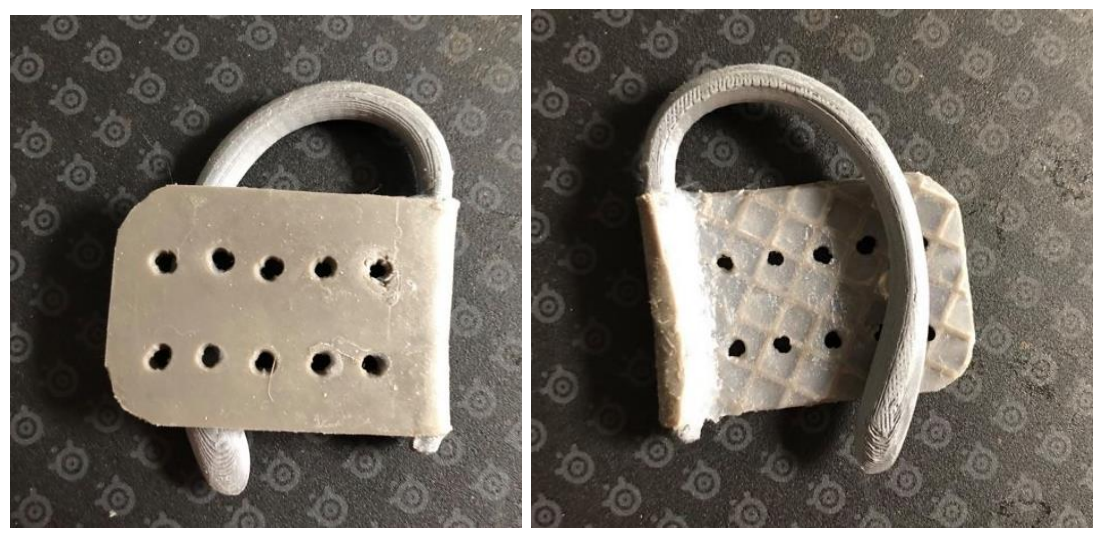

Figure 23. Earpiece.

Necklace: It is basically a base where users can attach the modules on it and wear as a necklace (Figure 23, 29). It has a tube which is TPE and holds the rope for a necklace. Its base is rubber and three modules can fit on it.

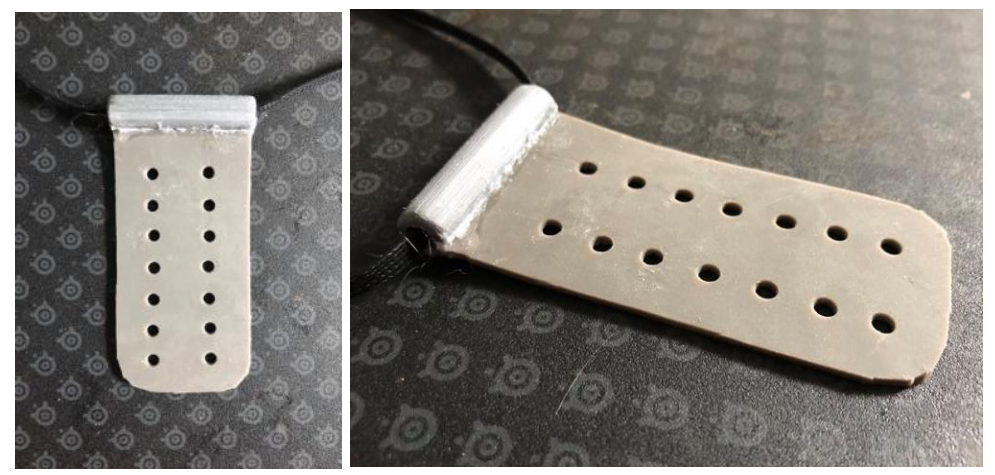

Figure 24. Necklace.

\subsubsection{Feedback Modules}

We designed three modules to house different electronics that can provide three types of feedback: visual, haptic and audio.. The size of the modules takes into consideration components dimensions and fit and are 3D printed. They are made of TPE.

Visual: The visual module has a 0.96 " OLED RGB display, it could display numbers and basic texts on it. It could be turned on and off with a button on the side. On the other side there is the socket to cable connection. Users can turn on the visual module with the button. They can see basic text and given numbers as their BLG (Figure 25, 28, 29). 

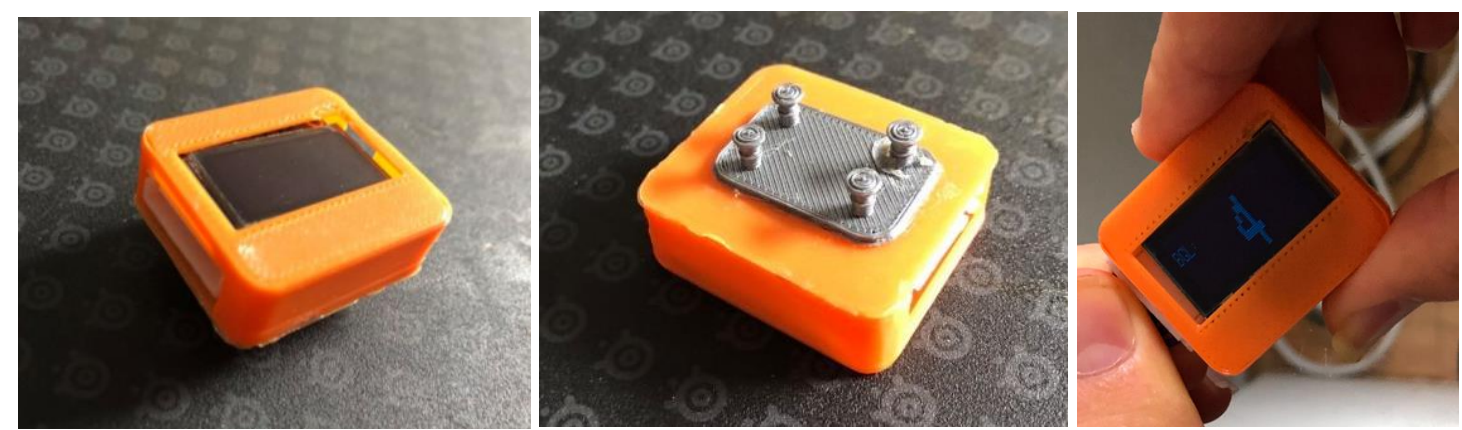

Figure 25. Visual feedback module.

Haptic: The haptic module has a vibration motor inside. It is turned off with a button. It also has a socket for cable connection. The threshold makes the motor work, and the user can turn it on with the button. It works after 10 seconds for a longer period. Users can again turn it off (Figure 26, 28, 29).
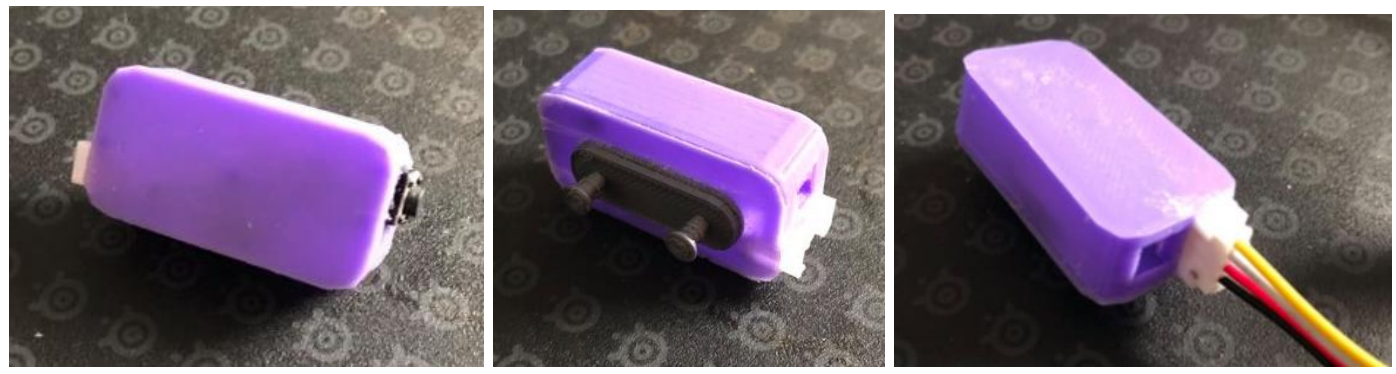

Figure 26. Haptic feedback module.

Audio: The audio module has an internal DC buzzer (Figure 27, 28, 29). A threshold code helped the buzzer work after the module connected to an electrical source. After the users turn the buzzer off, it starts buzzing again after 10 seconds for a longer period. Users can not adjust the sound level.
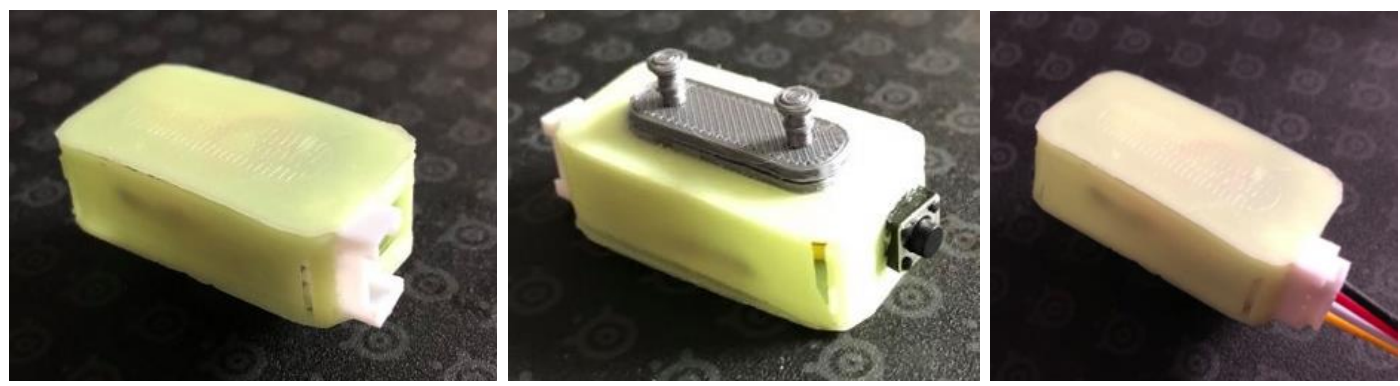

Figure 27. Audio feedback module.

\subsubsection{Implementation:}

DiaFit is operated and powered using a microcontroller (Arduino Nano 33 IoT) and a lithium ion polymer battery (3.7V 140mA). Modules are connected to the microcontroller using cable connections (Figure 25, 26, 27). An Arduino code sketch was developed to control the feedback mechanisms. The signal is filtered with a cut-off frequency of $3.3 \mathrm{~Hz}$. We created a threshold file to make the modules work. The code used for Arduino Nano is very simple. There are two conditionals written to make the circuit run and stop. The modules can be 
connected to the Arduino and controlled separately. Each module has strap pins on the back to enable connecting it to the accessories. They all can be used separately.

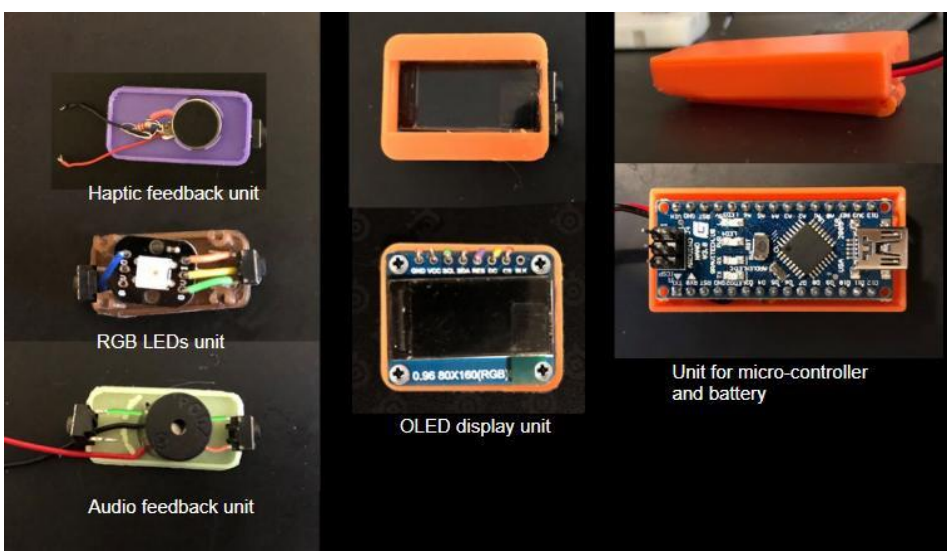

Figure 28. Components of the modules.

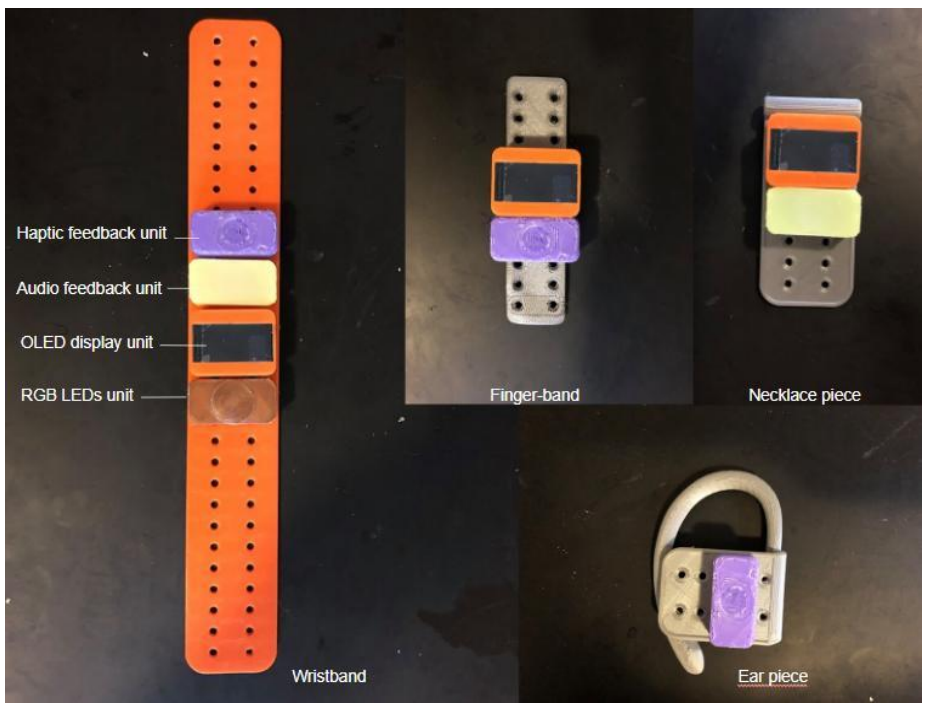

Figure 29. Sample configurations. 


\section{Evaluation}

We evaluated the DiaFit prototype with five participants. The aim of the evaluation session was to better understand how adults with Type 1 Diabetes view the process of building their own glucose monitors and whether people would even like to build such devices for themselves.

\subsection{Procedure}

We invited volunteer adult participants to a 30-45 minute interview and feedback session. Information about this study was advertised using our flyer and participant information sheet. If people were interested in participating, they emailed the researcher to book a suitable time for participation. Prior to the study, participants were asked to complete a short (5-6 mins) pre-study questionnaire that collected some basic background information. The sessions were video recorded and we took photographs of the generated prototypes to be used for data analysis. Participants interacted with the prototype by building a number of configurations of the glucose monitors and we asked them to think aloud and share their thoughts. At the end of the hands-on session, participants completed a short questionnaire about the usability of the prototype. Finally, we briefly discussed any final thoughts and questions they had.

\subsection{Participants}

Participants were invited on a first-come-first-served basis for this session. All five participants were 18+ years. Youngest participant was 20 years old and the oldest was 52 years old. One of them had participated in our previous interview and design sessions. All participants had T1D and had been using their T1D devices for a period of 5 to 30 years. In this chapter participants are referred to as "P" and given a number again. For example participant one is "P1" and participant number five is "P5". P3 and P5 considered themselves as makers. P1 was not sure if he was a maker or not but said he liked making digital applications and games on the computer. P3 said that he loved working with wood and metal as a hobby. He also had some experience developing diabetes tools and technologies. He liked trying to piece together things to help him better control his blood sugar levels. For example, he had a way to build a bluetooth radio frequency converter that allows people to know insulin level from an iphone. P5 was a design student. He was researching how to manage head injuries. P2 and P4 were not makers.

\subsection{Data Source and Analysis}

Data was collected via pre- and post-questionnaires and participants' feedback and interaction was video recorded. Post-study questionnaires included the heuristic evaluation questionnaire [74] and the TLX task analysis questionnaire [75]. Pre-study questionnaires collected demographic information and participants' views on creating artefacts by themselves. We first transcribed the interviews verbatim. We used a general inductive method in the analysis of the transcripts. Transcripts were read repeatedly and text segments coded for potential themes. Participants created a feedback module combination for each accessories and also selected aceesorrieswith combinations for five situational contexts. Themes that emerged and given combinations of accessories and modules for given contexts were used to inform the design and development of DiaFit. 


\section{Results}

Overall, all participants agreed on the importance of customization in the context of T1D. For example, P3 mentioned that he had previous experiences of customizing software for glucose monitors (using Loop, a diabetes management app) and thought that customization is important for diabetics. He said, "I think many of us would love to have some level of customization around all short of little things, from the way it designed, how it is worn to how it is worn or the type of data they are giving us. visual, audio, phone based, computer based. It is really important". P4 also expressed similar sentiments and said "it is an important feature for products. It gives flexibility and personal input and makes a product unique." Below we present participants responses and reactions related to the individual design features supported by DiaFit.

\subsection{Building Multiple Glucose Monitor Configurations}

Participants examined each of the accessories and feedback modules and considered how, when and where each might be used. Each participant built four different glucose monitor configurations using the accessories and modules (Figure 30).

In our procedure, we introduced the accessories and modules and described how participants may turn on the modules, attach them, control them, and wear them. We gave the participants time to interact and play with the accessories and modules. All the participants were interested in trying the accessories, attaching the modules. They interacted the modules for about 5 minutes. Connecting the modules to the main board was somewhat challenging because of the cables. Having experienced the feedback from modules participants shifted their attention to creating combinations for the accessories. P4 kept wearing the wristband during the interview. P5 was more interactive with the accessories, trying them while he was talking with me. Participants needed practice to mount the modules on the accessories and the cables on the modules. They found it challenging to attach the wristband and the ring with just one hand. 


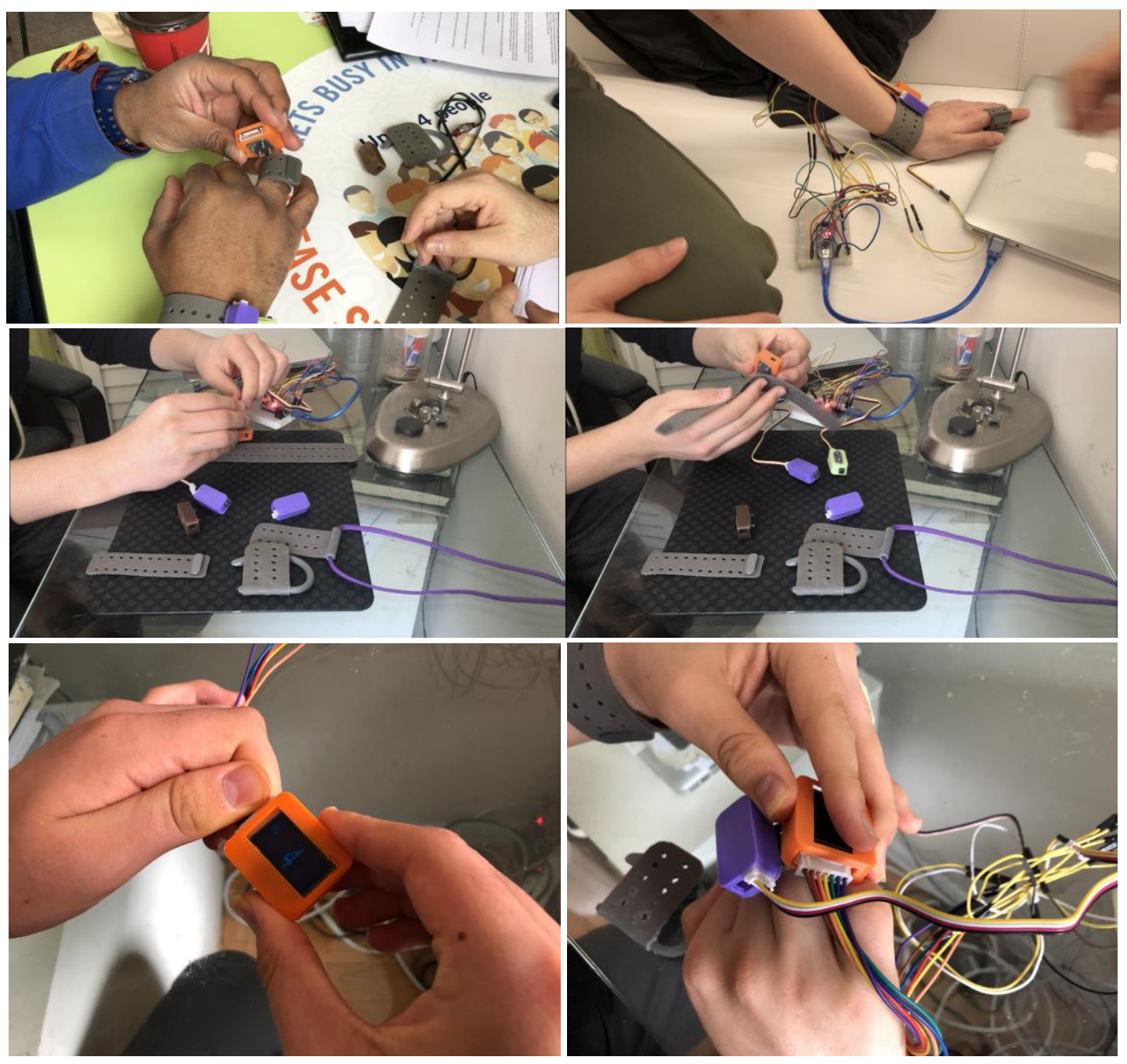

Figure 30. Users' interaction with the prototype. 
Table 1 shows the configurations built by each participant using different feedback modules per accessory.

Table 1. Configurations of modules per accessory.

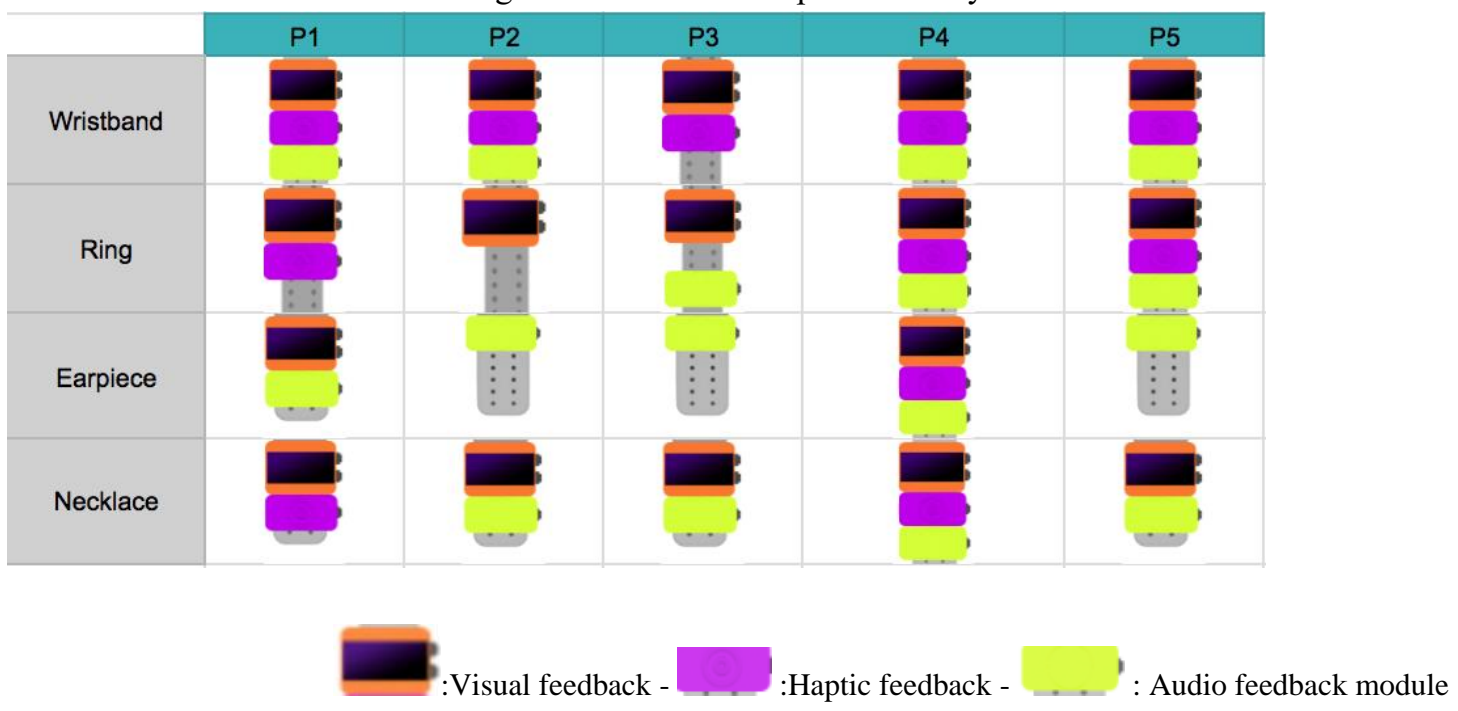

\subsubsection{Wristband}

P1, P2, P4, P5 said that the wristband could be worn anytime and anywhere. They mentioned they could wear it at home, school, work, gym, during outdoor activities as well as at social gatherings such as eating at a restaurant. All participants preferred having visual and haptic feedback modules attached to their wristband, except P3, who did not want to attach an audio feedback module. He mentioned that for his use cases, he envisions that haptic feedback should suffice and there was no need for audio feedback.

\subsubsection{Ring}

The ring was preferred by P1, P3 and P5. P1 especially showed significant interest in using the ring. P1 chose the haptic and visual display for the ring. P2 thought audio and haptic sensors are larger tnad visual sensors so he wanted to eliminate haptic and audio modules. P2 chose only the visual feedback module. However, the real challenge would be visual sensors and components in reality. P3 thought the ring could work with either visual or auditory signals. P4 thought that a ring would not provide adequate sound or vibration. Nevertheless, he still preferred visual, haptic and audio feedback for the accessory. P5 also preferred these three modules on the ring.

\subsubsection{Earpiece}

The earpiece was preferred by P1, P3 and P5. Participants preferred its hands-free nature for activities such as sports. P2 said that he would not use the earpiece because it might call attention to itself and invite questions. P4 worried it might fall off or break in a physical activity. P1 picked audio and vibration. He also thought vibration could be useful when he turned off the sound sometimes. P2, P3 and P5 thought only audio feedback would be necessary for the earpiece. P4 suggested using voice comments on the earpiece, because it is impractical to use the screen and take the device from your ear. 


\subsubsection{Necklace}

The necklace was preferred by P3 and P5. Initially P3 said he would not use the necklace, but after talking about the situational contexts he thought it could be very useful indeed and he would wear it. P1 preferred to use haptic and visual for the necklace. He hesitated using audio because he would not like others to hear the alarm. P2, P3 and P5 thought visual and audio would be enough for the necklace. Because the necklace did not touch the skin all the time, vibration would not be reliable. P4 decided that the sound was most essential.

\subsection{Situational contexts}

We asked participants how they would like to use their wearables in varied situational contexts (e.g., at gym, an outdoor activity, work/school, home and in public). We provided a few scenarios, and we asked them to build a glucose monitor that they might use in these cases. They were free to choose any of the accessories or even build something completely new (such as create a new feedback module). All participants chose not to build any new combinations but selected from their previously built monitors.

We prepared a table for the accessories selected for various situational contexts. P1, P3 and P5 selected more than one accessories for some of the activities they mentioned. For example, P1 said he would wear the ring for most of the outdoor activities, but would wear the earpiece for cycling. We place them in the order they mentioned they would use more often.

Table 2. Configurations of modules for each accessories for situational contexts.

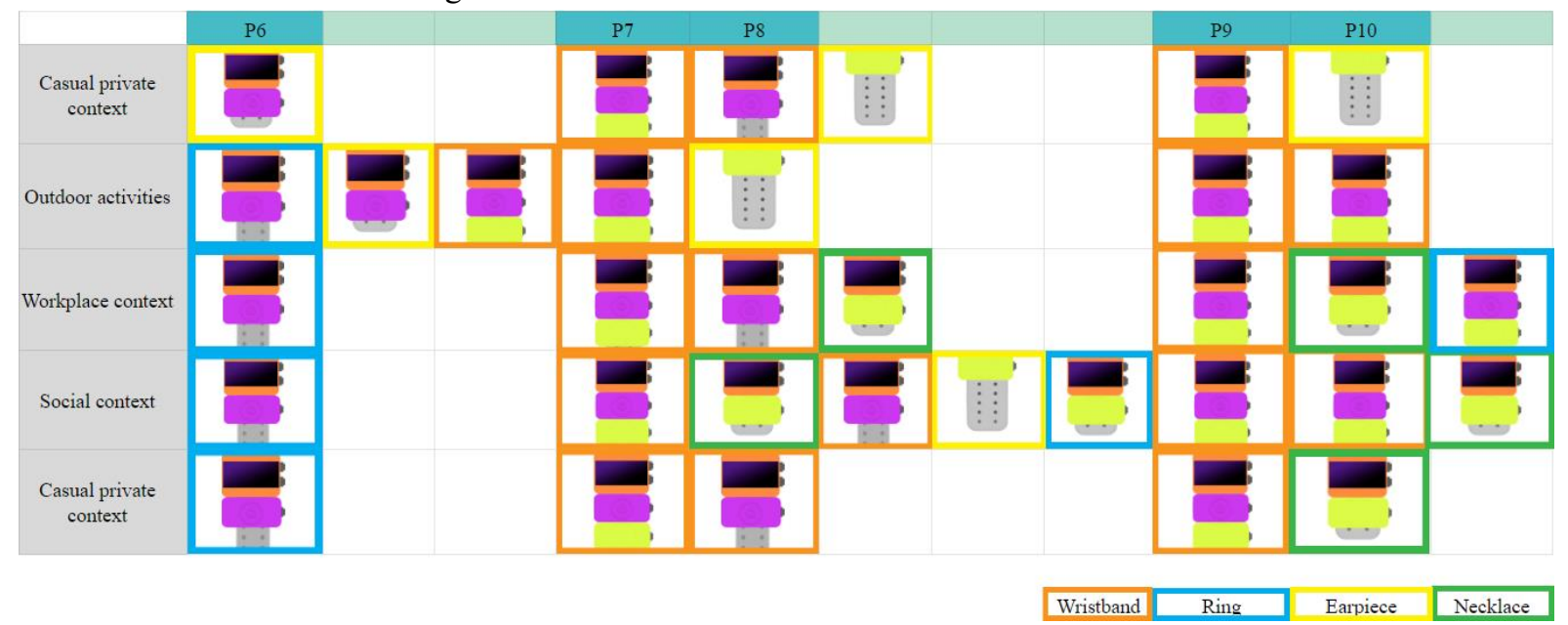

\subsubsection{Indoor activities}

The earpiece was one of the most common accessories selected for use in the gym and during outdoor sports. P1and P5 thought that the earpiece leaves his hands free during the time he is at the gym. He could use all the gym equipment and would not worry about missing a notification. P5 mentioned that the wristband may sometimes not be suitable, for example, while playing basketball, because of the attached modules. He thought the modules may get broken or he might hurt someone.P2 said that he could wear the wristband when he walks or plays sports. He mentioned he used to bike a lot before and wristbands 
would be his preference for riding as well. P3 said he would use the wristband and earpiece. P3 said, "I like the vibration. If you are at a gym, I have a very large range of glucose levels that I can not just worry about. But if I am critically low or I am drifting down I love a vibration. I think on the wrist or even in the ear would be great in the gym with vibration. I do not think I need audio because these are so close to the body, I can just feel it rather than hear it. Hearing may cause embarrassment. People around you may look at you, ask questions and it could be distracting. The vibration component is very innovative. I like it."

\subsubsection{Outdoor activities}

P1 said "A ring can be easily worn everywhere and anytime. After you get used to it, it won't be a problem to wear. It is also small and discreet. I can wear it when I play sports or camping. I feel comfortable among other people. I am not sure if it is possible but I would prefer my sensor on the ring. I wish the sensor was on the device." He mentioned more than one outdoor activity. He would prefer a ring if it is durable and the battery is long-lasting. He wanted the ring to be waterproof for water sports. When he is on a bike, an earpiece would be better because he could also communicate with other riders. He mentioned he would like voice comments to supply any notifications. He added using the wristband for trekking. He would not wear a necklace because of personal preference. P2 preferred wearing a wristband because it is easy to use and see the monitor. Monitor would be large enough to see the notes and colors. He wanted it to be waterproof for swimming and diving. P3 said that if you are doing a solitary activity and not in a crowded area, auditory would be fine, because you will be the only one hearing it. It could be as loud as you wish. Audio and vibration together would be great for rock climbing or bike riding. He thought it would be really fun and useful because while cycling, bike riders concentrate on the road. They do not want to take their eyes off the road and do not want to look down at a device. If two people cycle together they communicate back and forth. Some people listen to music as well. Therefore, he thought it would fit in with the culture of cycling. He said it would not interfere with the helmet. P5 wanted to wear the earpiece for any kind of outdoor activities and playing basketball.

\subsubsection{Workplace context}

P1 liked the ring idea and wanted to wear it for almost any occasion except sports. "Because the wristband and the necklace would be under my clothes it would be difficult to get the feedback and see the glucose levels. I could hide the monitor easily because the ring is small and discreet." P2 thought the wristband was discreet enough and easy to see and control. It could be formal enough to wear at work. P3 preferred the wristband over the necklace. He decided he would not wear the ring or the earpiece. They could be distracting and wearing them for as much as 6 hours might be problematic. He also added that the wristband would be comfortable to wear for a full day. P3 said they liked the wristband because "it is something on you, you are wearing and you do not have to take so much notice looking at it. You would feel the vibration at work or when you are driving. It is there when it is at a critical high and low threshold". P5 thought the wristband and the necklace could call attention to themselves. If the users are at work or school they would not want other people to see what they were wearing. He preferred wearing the necklace or the ring because of privacy. 


\subsubsection{Social context}

P1 preferred the ring in any social settings. He preferred his device to be discrete and not seen by others. He thought it would be easy to carry, check his blood glucose level and receive feedback. P2 and P4 preferred wristbands and they thought the wristband would be appropriate and functional in any social context. P2 said "[...] If you are eating out with friends you may have to leave the table [to check your monitor]. Just seeing your number on the monitor or wristband you do not have to leave the table.[...] Wristband or watch make it very practical, because you already carry it with you." P3 would wear any of them because new technology always brought new discussion in the diabetics community. However, sometimes he prefers to hide his device. He said he might wear the necklace under his shirt. P5 said if he was with his colleagues or his boss he preferred to be formal, and he would wear the wristband. With his friends or girlfriend he would wear the necklace. If people knew about his condition he would not worry about others seeing his device. The necklace made him feel more comfortable. He would not like wearing watches or other accessories. He would not feel discomfort with the necklace, but it might be itchy with the wristband. P1 and P3 commented that a necklace is a fashion statement, women in particular might find it appealing. P3 said, "I imagine myself taking it out of a shirt and looking at it. I feel a little bit awkward about it. I may also worry about losing it."

\subsubsection{Casual private context}

P1 would wear the ring. If he got used to wearing a ring he would not take it off, expect for charging or in the shower unless it were waterproof. He would feel safer and would not worry about hypo or hyperglycemia. P2,P3 and P4 preferred the wristband. P5 thought the most comfortable accessory was the necklace. He thought he could wear the necklace all the time except when exercising. He added that he found the necklace most interesting because it was easy to hide. P5 added washing the dishes or eating could be other contexts in which to wear the earpiece and necklace.

\subsection{Frequency of customization}

We asked participants their expected frequency of customization with our prototype. We expected them to rethink the concept of customization after talking about their daily activities and various situational contexts. However, we also understood that it is hard to imagine and give a precise number of uses and customization before using the prototype in real life. P1 said he would change the accessories a few times a day. On the weekend he would make changes on accessories and modules 3-5 times. If he was staying at home or doing activities in which he could use the same accessories and modules, he would not customize at all. P2 was not very interested in customization,but might switch the modules on his chosen accessory, the wristband, 2-3 times a week for special events or activities. P4 and P5 thought they would need to change settings digitally 2-3 times a day, sometimes more, sometimes less. P3 would change accessories and modules a couple of times a day. He said he would change it everyday. Even for the same activity he would experiment with various accessories and modules.

\subsection{Suggestions for Future}

P1 said he had never thought a ring could be a device for monitoring. He liked the ring especially because it was small, discrete and easy to wear. "I like the options for accessories. Playing sports is very 
important to me. I can carry these monitors with me during biking and when I am at the gym." P3 liked the design and the flexibility of the design. He thought that modules could be attached onto any form of accessories. The design was flexible, very easy to put on and very interesting. Modularity provides that flexible usage. They could customize the feedback and use various elements in different scenarios. They kept users hands free which was important. They definitely helped people in the gym, playing sports and while working with hands. P3 would love to switch around and customize his device. He would get bored if he had one device for all situations. He liked the ability to rotate the modules for different days and reasons. "I would show it to others if I am happy about this product and customization." P4 said he liked using vibration and other feedback and the multi sensorial experience with the device. The screen was small but it could be sufficient. P5 also asked to make the modules smaller than current dimensions. Our accessories and modules could be used as fashion pieces. P5 though our accessories and modules could be small or big if they are fashion pieces. He thought women could be more interested in using rings as a wearable device than men. It would be discrete and looked like a ring but actually be a medical device. He thought other people might not question it. We think they may take attention even more because they look like fashion pieces. With modularity, with various interaction means, these devices could create different experiences.

Although P1 gave ideas about using feedback modules for each accessory, he recommended combining all feedback mechanisms in one module. P2, P5 agreed with P4 and said modules should be one unit. They said he would not change that much but would like to be able to control the module with an app. They also suggested making the modules smaller and more fashionable. P1 was not sure how to carry accessories through the day. He suggested making a kit for accessories.

P1 suggested adding LED color coded feedback to show notifications depending on his BGL. We are planning to use color in the interface and visual feedback for future studies. P1, P3 was not happy about the design of the prototype, and thought it was old fashioned and bulky. P3 thought everything should be miniaturized. He said he would be worried about the modules for being too delicate, fragile and bulky. P3 thought that he would not wear the ring that much because of the current shape of the prototype. He thought it would be too distracting to wear. Especially for rings, it was considered as a problem by P1, P2, $\mathrm{P} 4$. They mentioned that the small size might also cause problems, because if the attached monitor was also very small then it would be hard to see and battery life might be very limited. We think, even if it is not possible to add sensors or components now because of their sizes, they will be available in the near future. P5's grandparents also have diabetes and they have to make injections everyday. He felt the vibration would provide more useful feedback than sound. We think all users with different abilities and ages would benefit from DiaFit and consider extending the study for various users with different abilities for future studies.

P2 suggested changing the rubber wristband with a softer material or adding another layer of soft material underneath the rubber. P3's main objection to the wristband had been its possible constriction and irritation. The wristband should be easily adjustable, easy to put on and off. He said that it felt a little bit clumsy. Some of the design elements might have an impact on the comfort. Magnets would make it easy to take the modules on and off. 
We asked about the location of the sensors. Prefered location was on the upper arm. The second choice was the stomach. P1 and P3 suggested the sensor could be on the ring. P4 said a sensor could be on the wristband in the near future should technology permit. He showed us the back of his watch which had a heartbeat sensor that touched his skin. Because the sensor should not move or dislocate, participants hesitated to put a sensor on other locations. We think smaller sensors will be available in the near future and will allow us to integrate the sensors on the accessories and modules.

\subsection{Heuristic evaluation}

Heuristic evaluation is a usability method for finding usability problems in a design as part of our iterative design process. It involves seven questions (Table 3) to examine our design and assess its compliance with "heuristics," or recognized usability principles. It helped to prevent any failure post-release [76].

Participants were invited to evaluate our prototype using the heuristic evaluation method [76]. Participants were given four tasks as part of which they interacted with the prototype and its parts multiple times and we discussed how, where, when and why the prototype could be used. They created combinations of modules on the accessories for various situational contexts. Each participant used the prototype multiple times. As a part of a heuristic evaluation questionnaire, we also asked participants to discuss the usability problems and usefulness of the modules and accessories and any related issues. Five participants completed this post-questionnaire. At the end of all the tasks, participants responded to six questions focused on usability issues (Table 3). The purpose of this set of questions was to catch oversights before they became truly problematic and to assess the satisfaction of the users and to evaluate product usability. We used likert scale from 1 to 5 (where 1 meant completely inappropriate and 5 completely appropriate) and determined the mean values of the participants' responses. Mean value gives the average value and shows the overall rating of participants. We can use the values comparing which number they are on or closer. For example; if mean value is 5, it means it is completely appropriate; if it is 4 , it is appropriate. We could consider it more appropriate if the value is more than 4.5.

Table 3. Results of heuristic evaluation.

\begin{tabular}{|l|c|c|c|c|c|c|}
\hline & P1 & P2 & P3 & P4 & P5 & Mean Value \\
\hline Appropriateness of the visual feedback & 4 & 4 & 5 & 5 & 5 & $\mathbf{4 . 6}$ \\
\hline Appropriateness of the audio feedback & 4 & 3 & 5 & 5 & 5 & $\mathbf{4 . 4}$ \\
\hline Appropriateness of the haptic feedback & 5 & 3 & 5 & 5 & 5 & 4.8 \\
\hline Easiness of understanding the features of the accessories & 5 & 5 & 4 & 5 & 5 & 4.8 \\
\hline Easiness of understanding the features of the modules & 4 & 4 & 4 & 4 & 5 & $\mathbf{4 . 2}$ \\
\hline Consistency of design layout & 5 & 4 & 5 & 3 & 5 & $\mathbf{4 . 4}$ \\
\hline Usefulness of the design concept & 5 & 4 & 5 & 4 & 5 & $\mathbf{4 . 6}$ \\
\hline
\end{tabular}


While during the hands on exploration with the prototype visual feedback was selected 17 times and haptic 13 times, the questionnaire indicated the haptic feedback was actually preferred. Participants understood the features of the accessories more easily ( $M V=4.8)$ than modules (MV=4.2). They considered the consistency of design layout more than appropriate $(\mathrm{MV}=4.2)$. This means the form and aesthetics of the accessories and modules are consistent in design. In addition they were asked to evaluate the overall usefulness of the design concept. The mean value was 4.6 indicating that participants found this concept useful (Table 3).

\subsection{Task analysis (TLX):}

To assess how effectively participants performed while interacting with the prototype,the NASA Task Load Index (NASA-TLX) was added to our post questionnaire. We provided descriptions for each measurement on the questionnaire to help participants answer accurately. Participants rated the overall task using a 5-point Likert scale (from 1 being very low to 5 being very high) and we determined the mean values of the participants' responses. Mean value gives the average value and shows the overall rating of the participants. We can use the ratings similar to heuristic evaluation.

Table 4. Results of task analysis.

\begin{tabular}{|l|c|c|c|c|c|c|}
\hline & P1 & P2 & P3 & P4 & P5 & Mean Value \\
\hline Mental demand & 1 & 1 & 1 & 2 & 1 & $\mathbf{1 . 2}$ \\
\hline Physical demand & 1 & 1 & 2 & 3 & 2 & $\mathbf{1 . 8}$ \\
\hline Temporal demand & 1 & 1 & 1 & 1 & 3 & $\mathbf{1 . 4}$ \\
\hline Effort & 2 & 2 & 2 & 2 & 1 & $\mathbf{1 . 8}$ \\
\hline Frustration & 2 & 1 & 1 & 1 & 1 & $\mathbf{1 . 2}$ \\
\hline Overall performance & 5 & 4 & 5 & 5 & 5 & $\mathbf{4 . 8}$ \\
\hline
\end{tabular}

The first question was how much mental and perceptual activity was required to use this prototype. We wanted to learn how easy or demanding the use of the prototype was. The computed mean value is 1.2, indicating that the task was considered easy and was not mentally challenging. Next we asked how much physical activity was required in order to use our prototype i.e. was the usability of the prototype easy or demanding. The computed mean value is 1.8 indicating participants used the prototype easily. A fourth question addressed mental and physical effort while using and wearing the accessories and modules. An assigned value of 1.8 suggests that only modest effort was required. Fifth question asked whether participants felt irritated, stressed, or annoyed while using the prototype. The value of 1.2 indicates that they felt relaxed and comfortable during the task. A final question asked how successful the participants were in performing the task and if they were satisfied with their performance. The value of 4.8 indicates that they were satisfied with their performance with the prototype (Table 4). 


\section{Discussion}

Wearables for monitoring T1D are intended to be integrated into all aspects of one's daily life [2]. The users' perception of wearability depends on personal preferences and context of use. Participation in sports and other physical activities, as well as varied social contexts, provide extra challenges for design.

This research has pointed out the influence that context can have on adults' experience and practice of self-management of Type 1 Diabetes using wearable devices. Although current self-monitoring devices meet medical requirements, the physical and social contexts in which they are carried also often influence their use and are generally overlooked in the design of these products. This is an important concern for the designers of T1D devices and we suggest that co-design design methods that are beyond the scope of current medical usability standards should be considered. Although this research focussed on the design of diabetes specific wearable devices, these concerns carry over to other conditions, other medical devices, and perhaps beyond to other assistive technologies [2]. Despite the health concerns involved in not carrying and using their T1D devices, other considerations often influence how adults make daily use of them. Self-consciousness and a simple desire to avoid embarrassment can affect their choices. User experience needs to be accounted for in the design of T1D devices. Such issues as multi-sensorial feedback, form, and aesthetics need to be explored.

Adaptability also proved to be important. Our participants' requirements changed with context of use. A device with which they might feel perfectly comfortable at home might be considered inappropriate for wear in more socially complex environments. A device designed for evening dress might not be suitable for a business meeting. This provides an opportunity for multiple, modular, customisable devices which can be easily adapted for different occasions. Frequently, participants simply desired their wearable technology to be less visible, designed to look like an everyday piece of jewellery or easily hidden under items of clothing.

\subsection{Individual experiences and differences}

Throughout our research it became clear that a negative affective experience could cause the misuse, or even non-use, of T1D technology. These affective experiences were often highly individual. Feelings such as frustration, annoyance, embarrassment, and discomfort were brought up again and again, and often identified as influences which undermine best self-management practices. These experiences resulted in behaviour ranging from people leaving their monitors in their locker at the gym to concealing them on their person to the extent that they were almost inaccessible.

Aesthetic sensibilities also varied when it came to choices involving the use of wearable CGMs. As Pateman et al. (2018), we also think that aesthetics plays an important, and currently underappreciated, role in use and continued engagement, particularly when the context of use is considered (81). A matter as simple as colour could be an issue. Sometimes the T1D device was perceived to be a nuisance if one was dressing for a particular occasion. P4 from the first interview session observed that she'd like her device to match her outfit. Most commonly responses focussed on how the devices should look: subtle, slim, small, and fashionable. People's lifestyles massively impacted adoption and use and varied significantly. Hobbies and routines could also interfere with use. P5 from the first interview session didn't like to carry her monitor while gardening. Another participant felt it was too much trouble to carry their monitor on a short walk. The device was even seen as an encumbrance when driving. People told 
us very personal and individual stories that had influenced their current practices and at least one (P3 from the first interview session) explicitly expressed gratitude for general advances in technology that had progressed beyond dripping blood onto test strips to determine their glucose level.

The range of individual experiences and practices does not make for an easy one-size-fits-all solution for making T1D devices suitable for peoples' daily lives. For adults who prefer to hide their devices during their everyday routines, a device designed to be inconspicuous would be best. Our study shows that everyone wants to hide their devices, at least sometimes. Our accessories and modules could help people with discreet accessoires and feedback options.

Some diabetics are more open about their everyday use of their devices. Some invite others to ask questions. A couple of our participants said they saw open use of their devices as an opportunity to educate those unfamiliar with the management of diabetes. However, even these participants felt the DiaFit could be more attractively designed. However, we could add to the challenge by suggesting it be made more visible, so that it might be more easily retrieved if misplaced. Some athletes may wish the DiaFit could be made sturdier without adding to its bulk and like the idea of greater visibility hoping it might encourage their teammates and opponents to be a little more alert.

These devices or modules could also be shared or lent to someone else to monitor. Minors or newly diagnosed diabetics could benefit from sharing their modules. Some users may desire additional monitors and modules. P3 imagined a situation in which a child playing hockey is wearing a sensor which relays to his mother in the stands who is wearing a second module. She would be alert to the condition of her child who may be distracted by the game. P4 mentioned that she shared her data with her father and P5's sister could receive a warning if she had a problem with her BGL. Such interactions could be supported by sharing of the modules. Similar interactions were shown to be beneficial by Seyed et al. (2018) in a different context, who developed a reconfigurable smartwatch with two touch sensitive display faces. Its interactions were used for notifications, private viewing, task switching, and sharing one of the modules [84]. Sharing behaviours and associated problems merit further study.

It is impossible to design devices universally, to accommodate all user requirements. We know from our interviews that diabetics' ideal requirements for their devices can be very complex and sometimes even self-contradictory. Sometimes it might resemble a fashionable accessory and other times it should be small, subtle and easily concealed. In certain situations it should look like exactly what it is: a medical device. P3 from the first interview session said "If you faint from hypoglycemia, you want those around you to be alerted to your condition, embarrassment be damned." All of this presents a dilemma to designers. Many participants expressed a desire for devices that were smaller and more compact, but at the same time easy to use. Some participants voiced dissatisfaction with the alarms and feedback on their devices. The prototype offered visual, haptic, and audio modules which could be turned off and on and used in any combination. The visual module provided some basic text and numerical indications of glucose levels. This could be enhanced to include better graphics and colour coded LEDs, among other possibilities. The audio alerts consisted of a buzzer but a variety of ringtones which might signal different levels of concern might be added. The haptic feedback module simply vibrated. This could be augmented with sequences which would provide more detailed information and might eliminate the need to check the monitor. These additions would result in a more universal design. DiaFit could also be of service to more people who require assistive technology. The visually impaired and those suffering hearing loss might 
benefit as well. A visually impared user may like to use audio and haptic feedback. Earpiece or necklace could be better alternatives as accessories for them. An elderly may use the audio device louder to hear better with a necklace. Smart devices have become increasingly commonplace and raise extraordinary expectations. Participants recommended such additions to our device as touch screens, voice commands, apps, cameras, and the ability to track other health related data. We are aware that people are very adaptable and want to have current available products such as iwatch or their smartphones. Because they are familiar with these products, they want to have a similar interaction and ease of use. A participant who took part in both sessions twice suggested adding hologram (or AR), even recommending another module just for hologram feedback. Despite such extravagant requests, users must be included in the design process and given options to best serve their individual needs. We should take requests and concerns seriously and involve them in our notes. They may carry a potential even if there is not any similar view and patterns.

\subsection{Customization and DIY}

As discussed above, it is clear that a one-size-fits-all solution is not appropriate. Customization has the potential to help users better meet their needs [77]. DIY approach has implications for the design and evaluation of current and future wearable medical devices used in the self-management of diabetes. Engaging people with T1D in design probes, as well as conducting participatory design sessions and running a living lab in homes with DIY kits that emphasise user-driven design might provide a path forward. This could involve using rapid prototyping and open-source hardware and software in creating bespoke medical technology. Our participants expressed keen interest in the customization of accessories and modules. They created various combinations of different accessories, took an interest in making their own devices, and expressed a satisfaction in contributing to the project as a whole. One ambitious participant (P1 from evaluation session) with a skill set in computer programming voiced a desire to create his own medical device, but confessed he would be reluctant to use it himself. He thought medical devices such as GCM need to be tested and get its confirmation of reliability. He said a problem in a DIY device or its coding could be harmful for the users. Adults who self-identified as makers were particularly interested in using DiaFit and building different versions of the monitors more often. They were more interested in customizing the accessories and modules, not just controlling the feedback digitally. The self-identified makers often seemed to enjoy the customization process for its own sake.

A CGM provides crucial information. Any error can result in serious problems. Software, coding, and the connection of components are all complex. This complexity is a barrier for people who want to build a medical device. Participants were interested in making and customizing their own devices but acknowledged that while they felt competent to assemble the pieces, parts, and components into a device, they wouldn't feel comfortable if they had to start from scratch. Participants said they would be open to building and customizing a device for others and sharing their experience. This, of course, would require an understanding of the other users' needs and expectations.

In the evaluation session participants were invited to interact with the components and to build a device with the parts we had provided. Because we explained how to assemble the parts, how the device was meant to function, and how it could be worn, participants did not have much trouble building and wearing a device. Sometimes connections did not fit and modules did not work and attaching the wristband and the ring with one hand proved to be a struggle. Participants offered a few suggestions as to how we could 
make the process easier. Using magnets to attach the elements was one proposed solution. Despite these difficulties, in the TLX task analysis they indicated that they did not have any problem understanding the features of the device and found wearing the accessories and attaching the modules easy.

Modular design, or modularity in design, is one of our key practices [78]. We subdivided a system into smaller parts called feedback modules (visual, audio, and haptic) which could be independently created, modified, replaced or exchanged between a set of accessories (wristband, ring, earpiece, and necklace). In this context modularity is at the component level and has a single dimension, component slottability. If there were no fixed organization of slots into accessories we could call it multidimensional modularity [79]. Our prototype has a modular system with limited modularity using modular components. We provide a level of customization with this design approach and offer benefits such as reduction in cost, interoperability, shorter learning time, flexibility in design, non-generationally constrained augmentation or updating (a new solution is achieved by merely plugging in a new module), and exclusion. We provide these by using similar designed modules which are operated similarly, and could be built and fixed by makers. This modular system could be viewed as more complete or holistic design, whereas platform systems are more reductionist, limiting modularity to components [80]. Users could devise combinations to create a wearable considering their needs and expectations. For example, users might use all the modules on the wristband but might choose to use only the audio feedback module on the earpiece. They might later decide to simplify the wristband to include only the visual module. This modular system also allows us to improve the features of the device over time. Without any other change, users can acquire newly developed modules while continuing to use the modules and accessories they already have.

As a result of the growing availability of 3D printers, end users are increasingly taking on the role of designer and it is easy to see how customised accessories for commercial devices could be created by any user with access to this equipment. However, when considering end-user customisation, it is important to recognize that users are generally not trained designers, and while "one-size-fits-all" wearables may not be appropriate, users do need support when choosing, customising or designing their own solutions. Initial steps are already being taken in this direction, but future research should further invest in creating adequate support for end-user customisation of smart-wearables. We found that involving users in codesign sessions and evaluation of designs, using their custom prototype, was key for them to better understand their own needs and desires. After using prototypes and talking about them, participants were often open to concepts which they had initially dismissed. A man who said he'd never wear a necklace later acknowledged that he might sometimes find one useful. People need the chance to explore alternatives.

\subsection{Limitations}

We are aware of the limitations of the generated prototype. We know developing a medical device requires teamwork and could take years. We spent almost a month designing and creating the circuits with our limited skill set. The limited functionality and durability of the prototypes meant engagement levels for evaluation were less than ideal. Therefore, we recommend higher prototype fidelity to achieve more realistic results. Another limitation was conducting the evaluations in an interview room, leaving the participants to imagine how they might use the prototype. Ideally, if we gave the prototypes to the participants and let them use them in various situational contexts the results might be more detailed and exact. Although we were concerned about the aesthetics of our design, the components we used did not 
allow us to explore this aspect as much as we might have wished as we focussed on the functionality of our prototype. Ideally, when fabricated, all the components will be smaller and more compact. Smaller components will also allow for greater flexibility in design.

The number of participants was limited to five in both the interview/co-design session and the evaluation session. Limited time and budget were also challenges. The advent of the covid-19 pandemic during the evaluation period meant that many of the potential and previous participants cancelled their appointments. Because the evaluation was necessarily hands-on, we decided not to attempt remote prototype evaluation.

We had four female participants at the interview/co-design session. Our one male participant from that session joined our prototype evaluations. Therefore, the participants in the two sessions had little overlap. All the participants in the evaluation session were male, which didn't prevent some of them from expressing opinions about how women might feel about the device. 


\section{Conclusion}

Inclusive and iterative methods were employed to better understand the adoption and use of T1D technologies by adults. It was found that physical activities, situational contexts, varying environments, and personal differences had an impact on how this technology fit into people's lives. For medical purposes these devices generally function well, but non-medical considerations also often have an impact on their use. Factors ranging from privacy issues to aesthetics carry considerable weight. Co-design sessions were conducted to address some of these concerns. Using devices in social settings was a common concern. To offer only one example, privacy was compromised by audio feedback which was too loud; health could be compromised if it was not loud enough. Our customizable prototype offers participants, particularly makers, variety options to better adopt their devices to the requirements of any given moment. Participants experimented with various combinations of the modules and accessories. They expressed an interest in participating in the design and development of medical devices. Customizability and DIY deserve a central role in this process. To state the obvious, not all diabetics are alike. Our research shows the importance of using inclusive design methods to better accommodate the everyday, and often very personal, contexts which influence selfcare. Customization and DIY also allow the individual to play a more active role in their own treatment rather than simply following instructions. Our research focused on adults with diabetes and had a limited sample set, but its usefulness is broader, as wearable devices play an increasing role as healthcare moves away from clinical settings into diabetics' everyday lives. Wearable medical devices will continue to improve and their future development will nodoubt be accelerated as the sharing of open-source technology plays an ever larger role in DIY communities.

\subsection{Future Work}

\subsubsection{Future research opportunities}

I believe that I have not (and could not) completely include all the situational contexts, physical activities, physical environments and individual differences that influence the use of Type 1 Diabetes technologies. I think this is a step towards understanding the various needs and possible solutions, but further exploration is certainly required. In the immediate and short term, how people interact with the user interface holds intriguing possibilities. The extent to which self-consciousness was revealed to be a significant factor in adult diabetic behaviour likely plays an exaggerated role in adolescents with this condition and needs to be addressed.

\subsubsection{Future design opportunities}

Considering the feedback I received from the participants, I am planning to improve the design of the DiaFit. Different accessories for varying situational contexts will be included in my further design development. Users could select which accessories they might need and acquire additional accessories if the need arose. DiaFit could be offered as a kit as well. There could be flexibility in the placement of the modules which could be attached to all accessories. They should be secure on the accessories while still easy to attach and remove. As four of our participants recommended, the separate modules could be integrated into a single unit which could be controlled from the interface (Figure 31). Additional options 
might include a backup battery and an insulin case. DiaFit's interface merits particular attention. An OLED touch screen could provide more up-to-date interaction and greater flexibility in interface design. While DiaFit is a DIY device, it need not look amateurish. Its components offer the maker the opportunity to create a compact and attractive wearable.

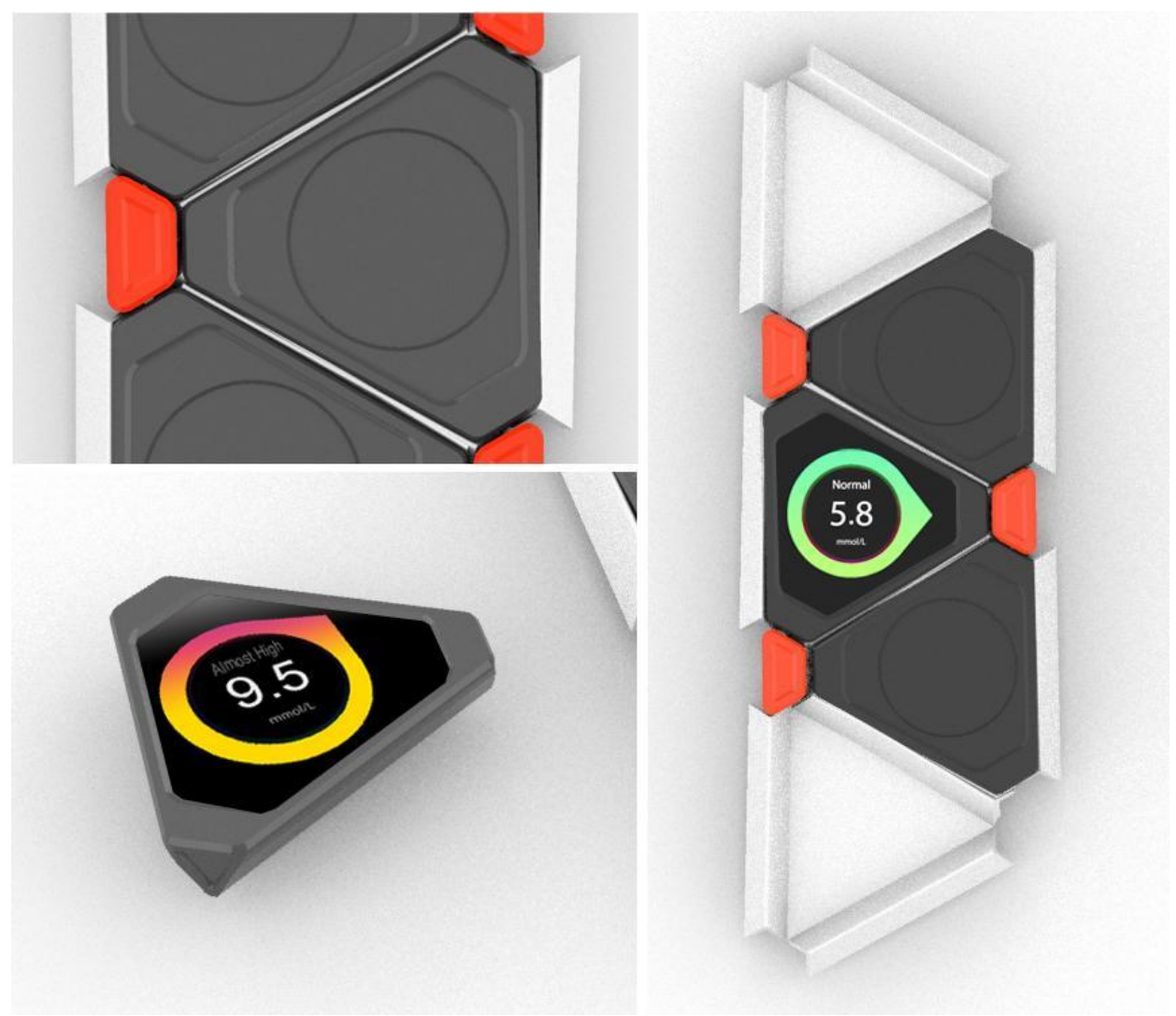

Figure 31. A digital sketch for DiaFit. 


\section{References}

1. O'Kane, A. A., Rogers, Y., \& Blandford, A. (2015). Concealing or revealing mobile medical devices?: Designing for onstage and offstage presentation. Paper presented at 1689-1698. doi:10.1145/2702123.2702453

2. O'Kane, AA; (2016) Individual differences and contextual factors influence the experience and practice of self-care with type 1 diabetes technologies. Doctoral thesis , UCL (University College London)

3. Rashotte, J., Tousignant, K., Richardson, C., Fothergill-Bourbonnais, F., Nakhla, M. M., Olivier, P., \& Lawson, M. L. (2014). Living with sensor-augmented pump therapy in type 1 diabetes: adolescents' and parents' search for harmony. Canadian journal of diabetes, 38(4), 256-262.

4. O'Kane, A. A., Hurst, A., Niezen, G., Marquardt, N., Bird, J., \& Abowd, G. (2016). Advances in DIY health and wellbeing. Paper presented at the 3453-3460. doi:10.1145/2851581.2856467

5. Gilmore J., Pine,B., J. (1997). The four faces of mass customization. Harvard business review 75, 91-101.

6. Sampada Marathe and S Shyam Sundar. 2011. What drives customization?: control or identity?. In Proc. CHI '11. ACM, 781-790.

7. S Shyam Sundar, Jeeyun Oh, Saraswathi Bellur, Haiyan Jia, and Hyang-Sook Kim. 2012. Interactivity as self-expression: a field experiment with customization and blogging. In Proc. CHI '12. ACM, 395-404

8. R.Gulotta, J. Forlizzi, R.Yang, and M.Wah Newman. 2016. Fostering Engagement with Personal Informatics Systems. In Proceedings of the 2016 ACM Conference on Designing Interactive Systems (DIS '16). ACM, New York, NY, USA, 286-300. DOI: http://dx.doi.org/10.1145/2901790.2901803

9. D.Harrison, P.Marshall, N.Bianchi-Berthouze, and J. Bird. 2015. Activity tracking: barriers, workarounds and customisation. In Proceedings of the 2015 ACM International Joint Conference on Pervasive and Ubiquitous Computing (UbiComp '15). ACM, New York, NY, USA, 617-621. DOI: http://dx.doi.org/10.1145/2750858.2805832

10. Eng, D. S., \& Lee, J. M. (2013). The promise and peril of mobile health applications for diabetes and endocrinology. Pediatric diabetes, 14(4), 231-238.

11. Chomutare, T., Fernandez-Luque, L., Årsand, E., \& Hartvigsen, G. (2011). Features of mobile diabetes applications: review of the literature and analysis of current applications compared against evidence-based guidelines. Journal of medical Internet research, 13(3), e65.

12. El-Gayar, O., Timsina, P., Nawar, N., \& Eid, W. (2013). Mobile applications for diabetes selfmanagement: status and potential. Journal of diabetes science and technology, 7(1), 247-262.

13. Canadian Diabetes Association. (2008). 2008 clinical practice guidelines for the prevention and management of diabetes in Canada.

14. NightsScouts (October 13,2016). What is the Nightscout project? Retrieved from: http://www.nightscout.info/ and https://www.youtube.com/watch?v=8g01XMXX43k\&feature=emb_logo

15. Meeri K. (December 6, 2019). DIY diabetes tech gains popularity with patients and parents fed up with clunky mainstream medical devices. Washington Post. Retrieved from https://www.washingtonpost.com/health/fed-up-with-clunky-diabetes-machines-do-it-yourselfers- 
re-engineer-devices-and-create-apps-and-software/2019/12/13/3f7c4e20-16c4-11ea-91103b34ce1d92b1_story.html

16. Cafazzo, J. A., Casselman, M., Hamming, N., Katzman, D. K., \& Palmert, M. R. (2012). Design of an mHealth app for the self-management of adolescent type 1 diabetes: A pilot study. Journal of Medical Internet Research, 14(3), e70. doi:10.2196/jmir.2058

17. Waite M, Martin C, Franklin R, Duce D, Harrison R. (2018) Human Factors and Data Logging Processes With the Use of Advanced Technology for Adults With Type 1 Diabetes: Systematic Integrative Review. JMIR Hum Factors 2018;5(1):e11, URL: https://humanfactors.jmir.org/2018/1/e11

18. Vincent, C. J., Niezen, G., O'Kane, A. A., \& Stawarz, K. (2015). Can standards and regulations keep up with health technology?. JMIR mHealth and uHealth, 3(2), e64.

19. Arduser, L. (2018). Impatient patients: a DIY usability approach in diabetes wearable technologies. Communication Design Quarterly Review, 5(4), 31-39. (NIGHTSCOUTs)

20. Reidy, C., Bracher, M., Foster, C., Vassilev, I., \& Rogers, A. (2018). The process of incorporating insulin pumps into the everyday lives of people with Type 1 diabetes: A critical interpretive synthesis. Health Expectations, 21(4), 714-729.

21. Webster, M., Foster, E., Comber, R., Bowen, S., Cheetham, T., \& Balaam, M. (2015, June). Understanding the lived experience of adolescents with type 1 diabetes: opportunities for design. In Proceedings of the 14th International Conference on Interaction Design and Children (pp. 140-149).

22. McCarthy, G. M., Rodriguez Ramírez, E. R., \& Robinson, B. J. (2017, June). Participatory design to address stigma with adolescents with type 1 diabetes. In Proceedings of the 2017 Conference on Designing Interactive Systems (pp. 83-94).

23. O'Kane, A. A., Hurst, A., Niezen, G., Marquardt, N., Bird, J., \& Abowd, G. (2016, May). Advances in DIY Health and Wellbeing. In Proceedings of the 2016 CHI Conference Extended Abstracts on Human Factors in Computing Systems (pp. 3453-3460).

24. O'Kane, A. A., Rogers, Y., \& Blandford, A. E. (2015, April). Concealing or revealing mobile medical devices? Designing for onstage and offstage presentation. In Proceedings of the 33rd annual ACM conference on human factors in computing systems (pp. 1689-1698).

25. Kickstarter (2019). Blocks. Retrieved from: https://www.kickstarter.com/projects/2106691934/blocks-the-worlds-first-modular-smartwatch

26. http://www.tuvie.com/sub-scope-glucose-monitoring-system-targets-type-1-diabetes-patient-thatplays-sports/

27. http://www.tuvie.com/monitoring-your-glucose-levels-is-easier-with-sugar-glucose-watch/

28. http://www.tuvie.com/armc-wearable-artificial-pancreas-concept-for-diabetics-patients/

29. https://designawards.core77.com/Consumer-Product/49516/Flore-The-Wearable-for-all-Diabetics

30. Selam, J. L. (2010). Evolution of diabetes insulin delivery devices.

31. American Diabetes Association. (2019). 7. Diabetes technology: standards of medical care in diabetes-2019. Diabetes Care, 42(Supplement 1), S71-S80.

32. Vechev, V., Dancu, A., Perrault, S. T., Roy, Q., Fjeld, M., \& Zhao, S. (2018, May). MoveSpace: on-body athletic interaction for running and cycling. In Proceedings of the 2018 international conference on advanced visual interfaces (p. 28). ACM.

33. Borges, N., Driller, M., Plews, D. (2016) Evaluating a new wearable lactate threshold sensor in recreational to highly trained cyclists. Sports Engineering, 19 (4), 229-235 
34. Sanders, E. B. N., \& Stappers, P. J. (2008). Co-creation and the new landscapes of design. Codesign, 4(1), 5-18

35. Blevis E., Lim Y., Roedl D., Stolterman E. (2007) Using Design Critique as Research to Link Sustainability and Interactive Technologies. In: Schuler D. (eds) Online Communities and Social Computing. OCSC 2007. Lecture Notes in Computer Science, vol 4564. Springer, Berlin, Heidelberg

36. Kessler, M. M. (2016). Wearing an ostomy pouch and becoming an ostomate: A kairological approach to wearability. Rhetoric Society Quarterly, 46(3), 236-250. doi:10.1080/02773945.2016.1171693

37. Teston, C. (2016). Rhetoric, precarity, and mHealth technologies. Rhetoric Society Quarterly, 46(3), 251-268. http://dx.doi.org/ 10.1080/02773945.2016.1171694

38. Blumenthal, S. (2015, September 1). Advancing health with information technology in the 21 st Century." The Huffington Post, Retrieved from http://www.huffi ngtonpost.com/susanblumenthal/advancing-health-with-inf_b_7968190.html

39. Luca, L., Dupont, B.,G.,R., Doungmene, F., Marken, F., Tyrrell, R., Guy, R., Illie, A. (2018). Non-invasive, adhesive patch promises measurement of glucose levels through skin without finger-prick blood test. Retrieved from nature.com/articles/doi:10.1038/s41565-018-0112-4

40. Williams, D. (18 March, 2018). No more needles: Diabetics can now monitor blood sugar levels through tears or sweat with a new biosensor. Nature News. Retrieved from https://www.naturalnews.com/2018-03-18-diabetics-monitor-blood-sugar-levels-through-tears-orsweat-with-a-new-biosensor.html

41. Kiera Roberts, Adam Jaffe, Charles F. Verge and Paul S. Thomas. Noninvasive monitoring of glucose levels: is exhaled breath the answer?. Journal of diabetes science and technology, (2012), 6(3), 659-664

42. Brian K. Smith, Jeana Frost, Meltem Albayrak and Rajneesh Sudhakar. Integrating glucometers and digital photography as experience capture tools to enhance patient understanding and communication of diabetes self-management practices. Personal and Ubiquitous Computing, (2007), 11(4), 273- 286.

43. Holubová, A., Vlasáková, M., Mužík, J., \& Brož, J. (2019). Customizing the types of technologies used by patients with type 1 diabetes mellitus for diabetes treatment: Case series on patient experience. JMIR mHealth and uHealth, 7(7), e11527. doi:10.2196/11527

44. Buckingham, B. (2008). Clinical overview of continuous glucose monitoring.

45. Diabetes Research in Children Network (DirecNet) Study Group, Buckingham B, Beck RW, Tamborlane WV, Xing D, Kollman C, Fiallo-Scharer R, Mauras N, Ruedy KJ, Tansey M, Weinzimer SA, Wysocki T. Continuous glucose monitoring in children with type 1 diabetes. J Pediatr. 2007;151(4):388-93.

46. Messer, L. H., Johnson, R., Driscoll, K. A., \& Jones, J. (2018). Best friend or spy: A qualitative meta-synthesis on the impact of continuous glucose monitoring on life with type 1 diabetes. Diabetic Medicine, 35(4), 409-418. doi:10.1111/dme.13568

47. Iscoe KE, Campbell JE, Jamnik V, Perkins BA, Riddell MC. Efficacy of continuous real-time blood glucose monitoring during and after prolonged high-intensity cycling exercise: spinning with a continuous glucose monitoring system. Diabetes Technol Ther. 2006;8(6):627-35

48. Tsalikian E, Mauras N, Beck RW, Tamborlane WV, Janz KF, Chase HP, Wysocki T, Weinzimer SA, Buckingham BA, Kollman C, Xing D, Ruedy KJ, Diabetes Research In Children Network 
(DirecNet) Study Group. Impact of exercise on overnight glycemic control in children with type 1 diabetes mellitus. J Pediatr. 2005;147(4):528-34.

49. Owen, T., Buchanan, G., and Thimbleby, H. Understanding user requirements in take-home diabetes management technologies. In Proc. BCS HCI, British Computer Society (2012), 268273.

50. Ritholz, M. D., Atakov-Castillo, A., Beste, M., Beverly, E. A., Leighton, A., Weinger, K., \& Wolpert, H. (2010). Psychosocial factors associated with use of continuous glucose monitoring. Diabetic Medicine, 27(9), 1060-1065.

51. Wysocki, T., Hirschfeld, F., Miller, L., Izenberg, N., Dowshen, S. A., Taylor, A., ... \& Kowal, K. (2016). Consideration of insulin pumps or continuous glucose monitors by adolescents with type 1 diabetes and their parents: stakeholder engagement in the design of web-based decision aids. The Diabetes educator, 42(4), 395-407.

52. Rashotte, J., Tousignant, K., Richardson, C., Fothergill-Bourbonnais, F., Nakhla, M. M., Olivier, P., \& Lawson, M. L. (2014). Living with sensor-augmented pump therapy in type 1 diabetes: adolescents' and parents' search for harmony. Canadian journal of diabetes, 38(4), 256-262.

53. O'Kane, A.A., Mentis, H.M. and Thereska, E. (2013). Non-static nature of patient consent: shifting privacy perspectives in health information sharing. In Proc. CSCW 2013, ACM Press (2013), 553-562.

54. McMahon, B.T., West, S.L., Mansouri, M., and Belongia, L. Workplace discrimination and diabetes: the EEOC Americans with Disabilities Act research project. Work: A Journal of Prevention, Assessment and Rehabilitation 25, 1 (2005), 9-18.

55. Owen, T., Buchanan, G., and Thimbleby, H. Understanding user requirements in take-home diabetes management technologies. In Proc. BCS HCI, British Computer Society (2012), 268273.

56. Ritholz, M.D., Smaldone, A., Lee, J., Castillo, A., Wolpert, H., and Weinger, K. Perceptions of psychosocial factors and the insulin pump. Diabetes Care 30, 3 (2007), 549-54.

57. Barnard, K. D., Wysocki, T., Thabit, H., Evans, M. L., Amiel, S., Heller, S., ... \& Angela Consortium. (2015). Psychosocial aspects of closed-and open-loop insulin delivery: closing the loop in adults with Type 1 diabetes in the home setting. Diabetic Medicine, 32(5), 601-608.

58. Holubová, A., Vlasáková, M., Mužík, J., \& Brož, J. (2019). Customizing the types of technologies used by patients with type 1 diabetes mellitus for diabetes treatment: Case series on patient experience. JMIR mHealth and uHealth, 7(7), e11527. doi:10.2196/11527

59. Delgado, J., Blasco, J. R., Portoles, L., Ferris, J., Hurtos, E., \& Atorrasagasti, G. (2010). FABIO project: Development of innovative customized medical devices through new biomaterials and additive manufacturing technologies. Annals of DAAAM \& Proceedings, 1541-1543.

60. Pandey, G., \& Vora, A. (2019). Open Electronics for Medical Devices: State-of-Art and Unique Advantages. Electronics, 8(11), 1256.

61. Rindelaub, J. D., Baird, Z., Lindner, B. A., \& Strantz, A. A. (2019). Identifying extractable profiles from 3D printed medical devices. PloS One, 14(5), e0217137. doi:10.1371/journal.pone.0217137

62. Merle, A., Chandon, J., Roux, E., \& Alizon, F. (2010). Perceived value of the Mass-Customized product and mass customization experience for individual consumers. Production and Operations Management, 19(5), 503-514. doi:10.1111/j.1937-5956.2010.01131.x 
63. Bardzell, J., Bardzell, S., \& Toombs, A. (2014, April). " now that's definitely a proper hack" selfmade tools in hackerspaces. In Proceedings of the SIGCHI Conference on Human Factors in Computing Systems (pp. 473-476).

64. @WeAreNotWaiting, (2015). “One Group, Many Solutions” \#CGMintheCloud \#Nightscout \#WeAreNotWaiting \#PebbleTime \#AppleWatch \#Pebble. Tweet. (23 July 2015). Retrieved July 23, 2015 from https://twitter.com/WeAreNotWaiting/ status/624155264460066816

65. @ danamlewis. (2015). "What does a closed loop artificial pancreas look like?” (The size of your battery may vary :) ) \#DIYPS \#OpenAPS. Tweet. (17 Sept, 2015). Retrieved September 17, 2015 from https://twitter.com/danamlewis/status/6447000394665 12384.

66. Damiano, E.(14 July 2015). The bionic pancreas.TEDxSacramento. Retrieved from https://www.youtube.com/watch?time_continue $=568 \& \mathrm{v}=\mathrm{bZXmfTxd79Q}$

67. American Diabetes Organization, (2019). Continuous glucose monitors. Retrieved from http://main.diabetes.org/dforg/pdfs/2019/2019-cg-continuous-glucose-monitors.pdf

68. Mills, J., Bonner, A., \& Francis, K. (2006). The development of constructivist grounded theory. International journal of qualitative methods, 5(1), 25-35.

69. Macaulay, A., Jagosh, J., Pluye, P., Bush, P., \& Salsberg, J. (2013). Quantitative Methods in Participatory Research: Being sensitive to issues of scientific validity, community safety, and the academic-community relationship. Nouvelles pratiques sociales, 25(2), 159-172.

70. Charmaz, K., \& Belgrave, L. L. (2007). Grounded theory. The Blackwell encyclopedia of sociology.

71. Schuler, D., \& Namioka, A. (Eds.). (1993). Participatory design: Principles and practices. CRC Press, 211-221.

72. Loeb, S., Morris, M., Dynarski, S., Reardon, D., McFarland, D., Reber, S. (2017). Descriptive analysis in education: A guide for researchers. Retrieved from: https://files.eric.ed.gov/fulltext/ED573325.pdf

73. Woiceshyn, J., Daellenbach, U. (2018). Evaluating Inductive versus Deductive Research in Management Studies: Implications for Authors, Editors, and Reviewers. Qualitative Research in Organizations and Management: An International Journal. 13. 00-00. 10.1108/QROM-06-20171538.

74. Nielsen, J., \& Molich, R. (1990, March). Heuristic evaluation of user interfaces. In Proceedings of the SIGCHI conference on Human factors in computing systems (pp. 249-256).

75. Noyes, J. M., \& Bruneau, D. P. (2007). A self-analysis of the NASA-TLX workload measure. Ergonomics, 50(4), 514-519.

76. Interaction Design Foundation (n.d.) Heuristic Evaluation. Retrieved from: https://www.interaction-design.org/literature/topics/heuristic-evaluation

77. Gilmore, J. H., \& Pine, B. J. (1997). The four faces of mass customization. Harvard business review, 75(1), 91-102.

78. Bonvoisin, J., Halstenberg, F., Buchert, T., Stark, R. (2016) A systematic literature review on modular product design, Journal of Engineering Design, 27:7, 488-514, DOI:

10.1080/09544828.2016.1166482

79. Ossher, H., Ungar, D., \& Kimelman, D. (2015, March). Subjective, multidimensional modularity with korz. In Companion Proceedings of the 14th International Conference on Modularity (pp. 15-18). 
80. Interaction Design Foundation (n.d.). What is holistic design? https://www.interactiondesign.org/literature/topics/holistic-design

81. Pateman, M., Harrison, D., Marshall, P., \& Cecchinato, M. E. (2018, April). The role of aesthetics and design: wearables in situ. In Extended Abstracts of the 2018 CHI Conference on Human Factors in Computing Systems (pp. 1-6).

82. Muller, M. (1991). PICTIVE-an exploration in participatory design. Paper presented at the 225231. doi:10.1145/108844.108896

83. Mahadevan, K., Somanath, S., \& Sharlin, E. (2018, April). Communicating awareness and intent in autonomous vehicle-pedestrian interaction. In Proceedings of the 2018 CHI Conference on Human Factors in Computing Systems (pp. 1-12).

84. Seyed, T., Yang, X. D., \& Vogel, D. (2016, May). Doppio: A Reconfigurable Dual-Face Smartwatch for Tangible Interaction. In Proceedings of the 2016 CHI Conference on Human Factors in Computing Systems (pp. 4675-4686). 
Appendix A - Sketches
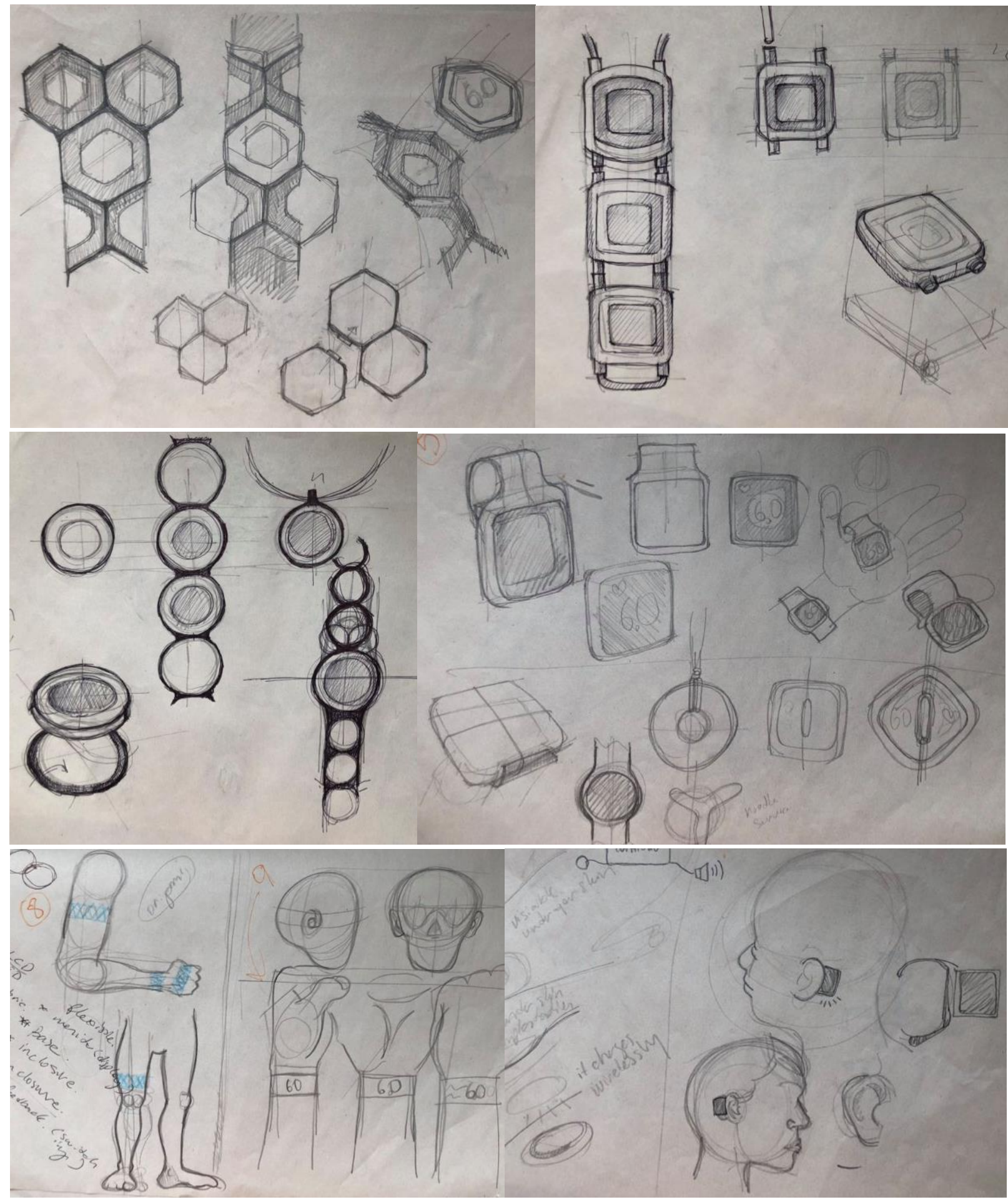


\section{Appendix B - Recruitment Poster}

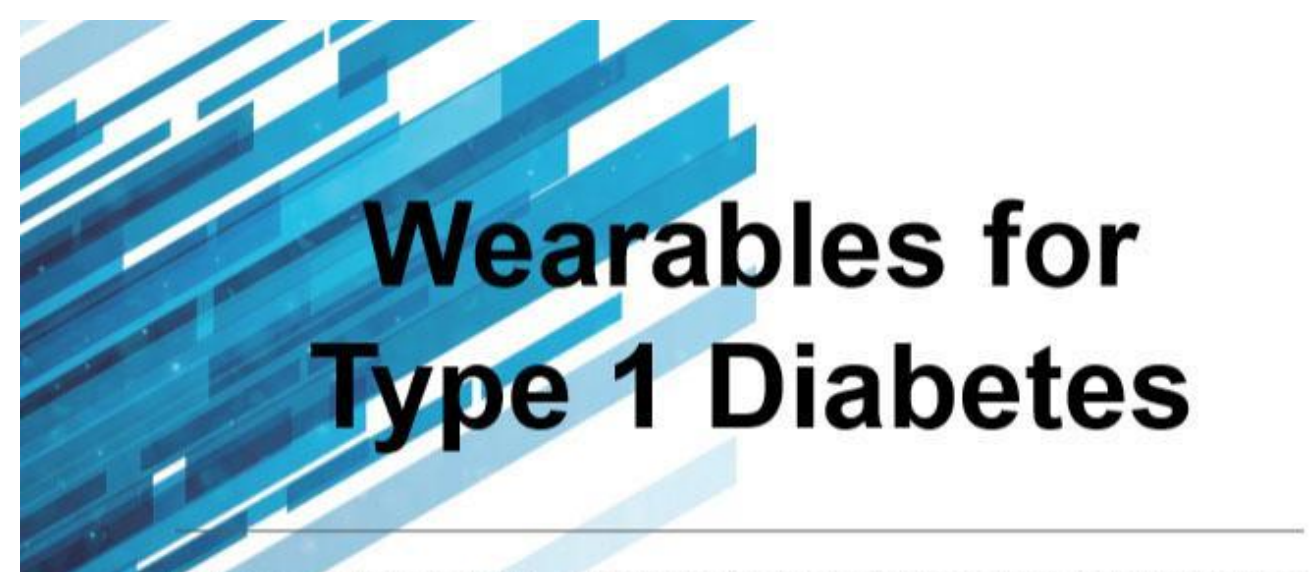

1 am a second year design student and I'm interested in understanding how people currently use their T1D medical devices during physical activities and if we can improve or enhance that experience. We built a prototype system consisting of electronics-modules that can be organized in a number of ways to build customized glucose monitors. We want to get feedback on our prototype and would love your help with that. If you are interested in participating, please see below:

\section{Who can participate?}

Anyone of age 18 years and above with Type 1 Diabetes can participate in this study. More specifically, we would love to talk to those who have had experience using their T1D devices when engaged in exercise or sports.

\section{What does it involve?}

You will take part in a 30-45 minutes session where we will interview and get feedback about our wearable prototype.

\section{When and Where?}

The study will take place during January 2020 and at OCADU 205 Richmond building

\section{Remuneration?}

As a thank you for your input and time we will give $\$ 10$ Amazon gift cards to our participants.

\section{More information?}

For more information, please contact Erman Akyol at: @student.ocadu.ca and Dr. Sowmya Somanath (Supervisor) at: @uvic.ca

Also see a more detailed info sheet at: http://bit.ly/30NSe1l

This study has been reviewed and received ethics clearance through the Research Ethics Board at OCAD University [101600]. If you have any comments or concerns, please contact the Research Ethics Office manager Christine Pineda,

\begin{tabular}{|l|l}
\hline O C & $\begin{array}{l}\text { Inclusive Design Program. Graduate Studies, OCADU } \\
\text { A D }\end{array}$ \\
& 205 Richmond St. West, 5th Floor Room 501 Toronto, ON \\
& Email: gradstudies@ocadu.ca Fax: 647-439-4194
\end{tabular}




\section{Appendix C - Heuristics Evaluation and Task Analysis of DiaFit}

\section{A. Background Information}

Participant:

Date:

\section{B. Users' Survey on DiaFit system based on heuristics principles}

1. Is the visual feedback given appropriate to learn about glucose levels?

Completely Inappropriate Acceptable Appropriate Very Inappropriate Appropriate

2. Is the haptic feedback given appropriate to learn about glucose levels?

Completely Inappropriate Acceptable Appropriate Very Inappropriate Appropriate

3. Is the audio feedback given appropriate to learn about glucose levels?

$$
\text { Completely Inappropriate Acceptable Appropriate Very Inappropriate Appropriate }
$$

4. It is easy to understand the features of accessories used throughout DiaFit. Disagree \begin{tabular}{lllll}
1 & 2 & 3 & 4 & 5 \\
\hline
\end{tabular}

5. It is easy to understand the features of modules used throughout DiaFit. Disagree $\begin{array}{llllll}1 & 2 & 3 & 4 & 5 & \text { Agree }\end{array}$

6. The same consistency of design layouts is used throughout DiaFit. Disagree $\begin{array}{llllll}1 & 2 & 3 & 4 & 5 & \text { Agree }\end{array}$

7. DiaFit is useful for me.

Disagree $\begin{array}{llllll}1 & 2 & 3 & 4 & 5 & \text { Agree }\end{array}$

C- Task Analysis: Task Load Index (TLX) method assesses the workload on five-point scales. Please scale the increments of high, medium and low estimates for each point result considering your customization experience with our prototype.

7- Mental Demand: How mentally demanding was the task?

$$
\text { Very Low } \begin{array}{llllll}
1 & 2 & 3 & 4 & 5 & \text { Very High }
\end{array}
$$

8- Physical Demand: How physically demanding was the task? Very Low $\begin{array}{llllll}1 & 2 & 3 & 4 & 5 & \text { Very High }\end{array}$

9- Temporal Demand: How hurried or rushed was the pace of the task? Very Low $\begin{array}{llllll}1 & 2 & 3 & 4 & 5\end{array}$ Very High

10- Effort: How hard did you have to work to accomplish your level of performance? Very Low $\begin{array}{llllll}1 & 2 & 3 & 4 & 5 & \text { Very High }\end{array}$

11- Frustration: How insecure, discouraged, irritated, stressed, and annoyed were you? Very Low $\begin{array}{llllll}1 & 2 & 3 & 4 & 5\end{array}$ Very High

12- Over all Performance: How hard did you have to work to accomplish your level of performance? Very Low $\begin{array}{lllllll}1 & 2 & 3 & 4 & 5 & \text { Very High }\end{array}$

Thank you for your help and participation 